\title{
Determination of Binding Constant and Stoichiometry for Antibody-Antigen Interaction with Surface Plasmon Resonance
}

\author{
Shiming Lin ${ }^{1,3, *}$ Adam Shih-Yuan Lee ${ }^{2}$, Chih-Chen Lin $^{2}$ and Chih-Kung Lee ${ }^{3}$ \\ ${ }^{1}$ Centre for Optoelectronic Biomedicine, National Taiwan University, Taipei, Taiwan; ${ }^{2}$ Department of Chemistry, Tamkang Univer- \\ sity, Tamsui, Taiwan and ${ }^{3}$ Institute of Applied Mechanics, National Taiwan University, Taipei, Taiwan
}

\begin{abstract}
A surface plasmon resonance (SPR) biosensor technology has recently been applied biochemically and clinically to the study of immunologic recognition and the evaluation of binding parameters for various interactions between antibodies (Abs) and antigens (Ags) at liquid-solid interface. The simple interaction between hapten and $\mathrm{Ab}$ fragment, e.g., variable single-chain fragment and antigenbinding fragment, can be described sufficiently by a 1:1 stoichiometry in SPR. However, the determination of the binding constant of an anti-protein $\mathrm{Ab}$ is usually complicated by the multivalence of the protein Ag. The SPR-based method enables direct determination of binding constants for a variety of specific $\mathrm{Ab}-\mathrm{Ag}$ interactions in real-time. It also allows estimation of the binding stoichiometry and binding ratio for low-, intermediate-, and high-affinity $\mathrm{Ab}-\mathrm{Ag}$ interaction systems. The present review is designed to indicate the theoretical background of SPR-based biosensor technology as well as to present the great variety of measurement modes of interaction kinetics that can be performed with these techniques. Quantitative aspects of the Ab-Ag interaction kinetics are reviewed, focusing especially on mono- and multi-valent $\mathrm{Ab}-\mathrm{Ag}$ interaction modes using a SPR biosensor. Four model binding systems developed recently for use with SPR biosenser are described with principles and examples: (i) one to one interaction mode, (ii) nonequivalent two-site interaction mode, (iii) multiple equivalent-site interaction mode and (iv) multisite interaction mode. This article closes with two descriptions of the determinations of the binding stoichiometry and maximum binding ratio of $\mathrm{Ab}-\mathrm{Ag}$ interactions.
\end{abstract}

Key Words: Monoclonal antibody, variable single-chain fragment, antigen-binding fragment, binding constant, affinity, dissociation constant, dissociation rate constant, association rate constant, stoichiometry, maximum binding ratio, surface plasmon resonance, resonance unit.

\section{INTRODUCTION}

The experimental determination of the binding constant (affinity) of an antibody $(\mathrm{Ab})$ for its antigen $(\mathrm{Ag})$ is of considerable importance. This is the basic experimental parameter in a variety of studies, such as the use of Abs as conformational probes (Goldberg, 1991), or in a thermodynamic approach to the molecular basis of $\mathrm{Ab}-\mathrm{Ag}$ interaction (Djavadi-Ohaniance and Friguet, 1992). Information obtained from binding stoichiometry allows one to measure the concentration of biologically active antigens (Fountoulakis et al., 1992; Biemann and Koshland, 1994) and to predict the binding topology. Also, it might help to localize the binding sites (Okun et al., 2001).

The single-chain antibody variable-region fragment $(\mathrm{scFv})$, the smallest construction of an $\mathrm{Ab}$ that retains the complete binding site, and the antigen-binding fragment $(\mathrm{Fab})$ from its monoclonal IgG preparation are monovalent and can combine with one haptenic Ag or with one separate epitope on the Ag molecule. The simplest case of $\mathrm{Ab}-\mathrm{Ag}$ interaction is that of the interaction of $\mathrm{scFv}$ (or Fab) $\mathrm{Ab}$ with monovalent $\mathrm{Ag}$, which includes truly monovalent haptenic $\mathrm{Ag}$ and a single, non-repeating site on the monomeric protein $\mathrm{Ag}$. One molecule of immunoglobulin G ( $\operatorname{IgG}$ ) monoclonal antibody $(\mathrm{mAb})$ has two identical combining sites and can combine with two separate homologous hapten molecules, with separate epitopes on two Ag molecules, or with two identical epitopes on one polymeric $\mathrm{Ag}$ molecule. Interactions between divalent mAb and monovalent $\mathrm{Ag}$ can be either cooperative or noncooperative: the binding at one site may or may not influence binding at another site. The monoclonal mAb preparation only contains one epitope specificity. However, the polyclonal $\mathrm{Ab}$ from immune serum contains a heterogeneous population of Abs which vary in concentration and in the strength (i.e., affinity) of their binding to the immunogen.

Based upon the Law of Mass Action, a variety of experimental approaches have been developed to facilitate the verification of an interaction and the calculations of binding parameters, such as the

*Address Correspondence to this author at the Center for Optoelectronic Biomedicine, National Taiwan University / College of Medicine, 1-1 Jen-Ai Road, Taipei 100, Taiwan; Fax: +886-2-23949125; E-mail: sml@ntumc.org dissociation constant, stoichimetry, and the kinetic constant for the interaction between di- or mono-valent $\mathrm{Abs}$ and mono- or multivalent Ags (Berzofsky and Berkower, 1983). Among the various existing methods for estimating the binding parameters, most demand separation of free and bound reactants (e.g., equilibrium dialysis, ultracentrifugation, ultrafiltration, chromatography, and planar electrophoresis). They involve addition of reactants, mixing, incubation, and the subsequent determination of the concentration of the reactants or products at time intervals thereafter. Such conventional techniques, which have been widely used for the study of $\mathrm{Ab}-\mathrm{Ag}$ interactions, can provide signals of individual interacting components (free $\mathrm{Ab}$ or $\mathrm{Ag}$ ) and/or antibody-antigen complexes (AbAg), thus avoiding the disturbances caused by the matrix of coexisting compounds. However, they are constrained by a number of problems, such as loss of reactant due to non-specific adsorption, volume shift, inapplicability to unstable reactants due to time consumed, Donnan effect, leakage of bound reactants through membranes, deviation of $\mathrm{Ab}-\mathrm{Ag}$ binding equilibrium, as well as estimation errors caused by viscosity, sedimentation, and back-diffusion (Liu et al., 1999).

Surface plasmon resonance (SPR) biosensor technology has been established as a commercial instrument for almost 15 years. SPR-based BIAcore ${ }^{\circledR}$ instruments have recently been used to measure a variety of biomolecular interactions in real time (Jonsson, 1992). It is capable of characterizing binding reactions without labeling requirements. In a typical SPR experimental set-up, incident light is reflected from the internal face of a prism in which the external face has been coated with a thin metal film (Fig. 1). At a critical angle, the intensity of the reflected light is lost to the creation of a resonant oscillation in the electrons at the surface of the metal film (Davies, 1994). Since the critical angle is dependent on the refractive index of the material present on the metal surface, this real-time method has been used to measure molecular interactions at solid-liquid interface. One reactant, referred to as the ligand, is immobilized to the sensor surface and the other reactant, referred to as the analyte, flows past this surface in solution. When the target and the probe interact to form a complex, a response is generated and monitored in real-time. SPR biosensors measure the change in refractive index in the vicinity of the surface of sensor chips to 
which ligands are immobilized (Markey, 1999). Because the changes in the refractive index are proportional to the changes in the adsorbed mass, the SPR technology allows detection of targets interacting with the probe immobilized on the sensor chip. It can thus be used to study the interactions of any biological system from proteins, oligosaccharides, oligonucleotides, and lipids to small molecules, viral particles, and bacterial cells. More fundamentally, the technique allows the direct observation of association and dissociation rate constants that contribute to the overall binding affinity (Lin et al., 2005).

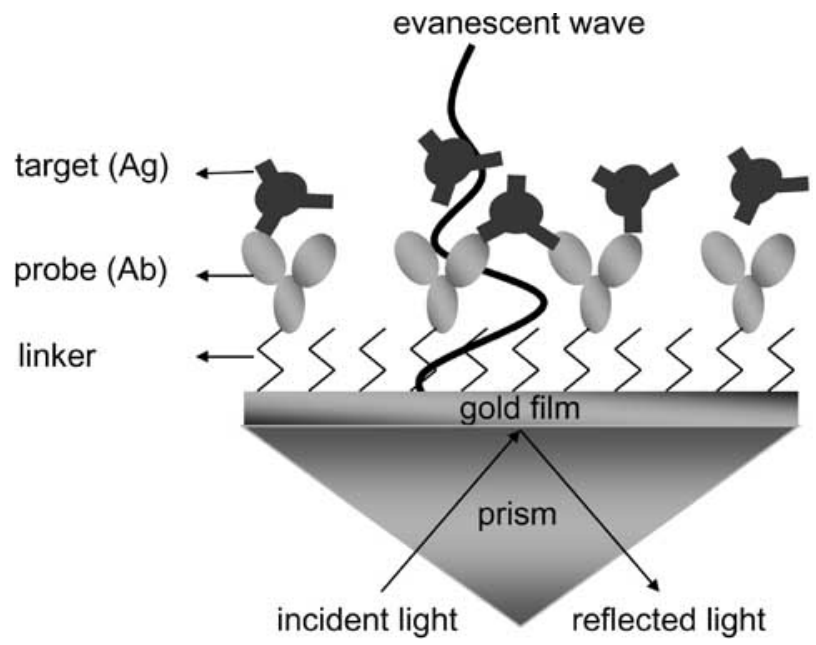

Fig. (1). In real-time biospecific interaction analysis, the binding events are detected using the optical phenomenon surface plasmon resonance, SPR. Under conditions of total internal reflection at a metal-coated interface, an evanescent wave propagates into the medium of low refractive index on the non-illuminated side. The SPR response is correlated to changes in refractive index at the sensor chip surface, caused by concentration changes on the sensor chip surface, e.g., when the target (Ag) binds to the immobilized probe $(\mathrm{Ab})$. The SPR signal is monitored continuously so that interactions between target and probe can be studied in real time.

The use of SPR for the measurement of binding parameters for interactions has been reported. Qualitative applications range from follow up of purification (Lackmann et al., 1996), epitope mapping (Myszka et al., 1999), ligand fishing (Rich and Myszka, 2000) to identifying small molecule leads in a screening mode (Markgren $e t$ al., 1999). Quantitative applications include measuring reaction kinetics $\left(k_{\mathrm{a}}, k_{\mathrm{d}}\right)$ (Morton and Myszka, 1998) and binding constants $\left(K_{\mathrm{D}}\right)$ (Myszka et al., 1998) and determining the active concentration of molecules (Zeder-Lutz et al., 1999). Thermodynamic information can be obtained by measuring reaction rates and equilibrium constants at different temperatures (Roos et al., 1998). When experiments are performed carefully, SPR biosensors can also be used to determine the binding stoichiometry and mechanism of the interaction (Morton and Myszka, 1998; Lin et al., 2005).

The recent development of SPR-based biosensor technologies has made it possible to directly monitor the binding of low molecular mass compounds to immobilized macromolecules and thus allowed the study of fast molecular interaction kinetics and the evaluation of binding constants for $\mathrm{Ab}-\mathrm{Ag}$ interactions in free solution (Table 1). In these reports, the simpler $\mathrm{Ab}-\mathrm{Ag}$ interaction can be sufficiently described by a $1: 1$ stoichiometry (Canziani et al., 2004; Son et al., 2004; Ja et al., 2005; Dubreuil et al., 2005). However, the determination of the binding constant for $\mathrm{Ab}-\mathrm{Ag}$ interaction is complicated by the multivalence (multiple binding-sites) of the $\mathrm{Ab}$ (or $\mathrm{Ag}$ ). When the $\mathrm{Ab}$ is monovalent, aggregates will not come up for a monovalent heptenic Ag. However, for (homo)polyvalent protein $\mathrm{Ag}$ (Gomes and Andreu, 2002; Herr et al., 2003; Tobita et al., 2004; Wittel et al. 2005; Yang et al., 2005); aggre- gates can be formed depending on conditions. The problem of aggregation can be minimized by the use of a very low concentration of reactants or by performing the determination with an analyte in excess. In addition, in some situations, the Ab (e.g., IgG and IgM) has two or multiple binding sites, each of which has a similar or different intrinsic affinity for binding independently to each $\mathrm{Ag}$ molecule (Herr et al., 2003; Tobita et al., 2004). It is not always easy to determine each of the intrinsic affinity. The problem of the heterogeneity of affinity of multivalent $\mathrm{Ab}$ (or $\mathrm{Ag}$ ) still remains an obstacle to meaningful interpretation of data obtained. The objective of the present review is to be a tutorial with up to date examples to illustrate. It starts with the one to one interaction analysis of $\mathrm{Ab}-\mathrm{Ag}$ interaction kinetics by SPR biosensor and follows with an in-depth description about the quantitative expression and evaluation of binding constants for $\mathrm{Ab}-\mathrm{Ag}$ interaction kinetics by SPR method, with particular emphasis on the non-cooperativity and cooperativity of one, two, and poly binding sites on the related $\mathrm{Ab}$ (or Ag).

\section{PRINCIPLE OF REAL-TIME Ab-Ag INTERACTION KI- NETICS}

In SPR biosensors, one of the reactants, e.g., Ab (probe), is immobilized on a sensor chip. The other reactant, Ag (target), enters at one end, is transported by diffusion and flow to the sensor surface, where it reacts with immobilized Ab (Fig. 2A). Change in the resonance signal expressed as resonance units (RU) are followed as a function of time and presented as a sensorgram (Fig. 2B). From this data binding kinetics of the $\mathrm{Ab}-\mathrm{Ag}$ interaction can be determined. The formation of surface-bound complex $\mathrm{AgAb}$ in the flow cell between target Ag and surface-bound probe Ab can be represented by the scheme

$$
\mathrm{Ag}_{\text {bulk }} \frac{k_{\mathrm{m}}}{k_{\mathrm{m}}} \mathrm{Ag}_{\text {surface }}+\mathrm{Ab} \frac{k_{\mathrm{a}}}{k_{\mathrm{b}}} \mathrm{AgAb}
$$

where $k_{\mathrm{m}}$ is the rate constant for mass transport to and from the surface (the rate constant for mass transport is the same in both directions) and $k_{\mathrm{a}}$ and $k_{\mathrm{d}}$ are the association and dissociation rate constant for the formation of $\mathrm{AgAb}$ complex, respectively.

In the ideal situation, neither the transport of Ag to the sensor surface nor its transport within the linker layer influences the binding kinetics. This happens when transport is fast compared to binding. In this case the $\mathrm{Ag}$ concentration rapidly becomes uniform in space and constant in time, equals to the injection concentration $[\mathrm{B}]_{\mathrm{o}}$ in the bulk phase, and the measured forward and backward rate constants approximate to the constants for interaction kinetics (Glaser, 1993). Under these conditions, the rate of complex formation may be described as:

$$
\mathrm{d}[\mathrm{AgAb}] / \mathrm{d} t=k_{\mathrm{a}}[\mathrm{Ag}]\left([\mathrm{Ab}]_{\mathrm{o}}-[\mathrm{AgAb}]\right)-k_{\mathrm{d}}[\mathrm{AgAb}]
$$

where $[\mathrm{AgAb}]$ is the amount of bound analyte, $[\mathrm{Ag}]$ is the amount of free $\mathrm{Ag},[\mathrm{Ab}]_{\mathrm{o}}$ is the total amount of $\mathrm{Ab}$ on the sensor surface. The on and off rates of binding are directly observable and the binding constants may be calculated by two methods described below.

\subsection{Equilibrium Analysis}

If the total amount of probe $[\mathrm{B}]_{\mathrm{o}}$ is expressed in terms of the maximum target binding capacity of the surface, i.e., the total amount of $\mathrm{Ab}$ binding-site on the sensor surface, all concentration terms can then be expressed as SPR response signal $R$, eliminating the need to convert from mass to molar concentration. Under pseudo first order conditions where the free target concentration is held constant in the flow cell, the binding can be described by Eq. [3]:

$\mathrm{d} R / \mathrm{d} t=k_{\mathrm{a}} C\left(R_{\max }-R\right)-k_{\mathrm{d}} R$ 
Table 1. SPR-Based Studies of Biospecific Interaction Analysis between Abs and Ags

\begin{tabular}{|c|c|c|c|c|c|c|}
\hline Target $^{\mathrm{a}}$ & Probe $^{b}$ & $K_{\mathrm{a}}\left(\mathbf{M}^{-1} \mathbf{s}^{-1}\right)^{\mathrm{c}}$ & $K_{\mathrm{d}}\left(\mathrm{s}^{-1}\right)^{\mathrm{d}}$ & $\mathbf{K}_{\mathbf{D}}(\mathbf{M})^{\mathrm{e}}$ & $n^{f}$ & Ref. \\
\hline $\begin{array}{l}\text { IgG1-Fc } \\
\text { sFc } \gamma \text { RIIb }\end{array}$ & $\begin{array}{l}\text { sFcyRIIb } \\
\text { IgG1-Fc } \\
\text { Intact IgG1 } \\
\text { R/A IgG1 }\end{array}$ & & & $\begin{array}{l}(0.98 \pm 0.01) \times 10^{-6} \\
(0.94 \pm 0.06) \times 10^{-6} \\
(0.95 \pm 0.02) \times 10^{-6} \\
(1.89 \pm 0.40) \times 10^{-6}\end{array}$ & $\begin{array}{l}1.00 \pm 0.04 \\
0.91 \pm 0.01 \\
0.97 \pm 0.04 \\
1.06 \pm 0.02\end{array}$ & [Mimura et al., 2001] \\
\hline Platelet-derived growth factor-BB (PDGF-BB) & basic fibroblast growth factor (bFGF) & $(1.27 \pm 0.06) \times 10^{6}$ & $(1.71 \pm 0.06) \times 10^{-2}$ & $(13.5 \pm 8.0) \times 10^{-9}$ & & [Russo et al., 2002] \\
\hline $\begin{array}{l}\text { C-propeptide domains of procollagens (CRI) } \\
\text { CRIII }\end{array}$ & $\begin{array}{l}\text { Procollagen C-Proteinase enhancer } \\
\text { (PCPE) }\end{array}$ & & & $\begin{array}{l}(1.69 \pm 0.23) \times 10^{-7} \\
(3.69 \pm 0.5) \times 10^{-7}\end{array}$ & $\begin{array}{l}1 \\
1\end{array}$ & [Ricard-Blum et al., 2002] \\
\hline $\begin{array}{l}\operatorname{IgG} 1, \kappa(33 \mathrm{D} 5) \\
\quad(37 \mathrm{E} 1) \\
\mathrm{IgG} 2 \mathrm{a}, \lambda(141 \mathrm{D} 4)\end{array}$ & StO4-BSA & $\begin{array}{l}1.8 \times 10^{5} \\
2.3 \times 10^{5} \\
1.2 \times 10^{6}\end{array}$ & $\begin{array}{l}1.0 \times 10^{-4} \\
1.9 \times 10^{-4} \\
3.9 \times 10^{-4}\end{array}$ & $\begin{array}{l}5.7 \times 10^{-10} \\
8.0 \times 10^{-10} \\
3.3 \times 10^{-10}\end{array}$ & & [Leickt et al., 2002] \\
\hline $\begin{array}{r}\text { FMDV antigenic peptide }(152 \mathrm{nM}) \\
\qquad \begin{aligned}(305 \mathrm{nM}) \\
(610 \mathrm{nM}) \\
(1220 \mathrm{nM}) \\
(2440 \mathrm{nM})\end{aligned}\end{array}$ & anti-FMDV mAb & $\begin{array}{l}6.0 \times 10^{4} \\
5.8 \times 10^{4} \\
5.9 \times 10^{4} \\
6.1 \times 10^{4} \\
6.2 \times 10^{4}\end{array}$ & $\begin{array}{l}2.4 \times 10^{-3} \\
2.6 \times 10^{-3} \\
2.6 \times 10^{-3} \\
2.7 \times 10^{-3} \\
2.9 \times 10^{-3}\end{array}$ & $\begin{array}{l}4.0 \times 10^{-8} \\
4.3 \times 10^{-8} \\
4.3 \times 10^{-8} \\
4.3 \times 10^{-8} \\
4.8 \times 10^{-8}\end{array}$ & & [Gomes and Andreu, 2002] \\
\hline $\begin{array}{l}\text { wild-type antigen of LFA-1 } \\
\text { locked open mutant antigen of LFA-1 }\end{array}$ & $\mathrm{HI} 111 \mathrm{mAb}$ & $\begin{array}{l}1.25 \times 10^{5} \\
2.32 \times 10^{4}\end{array}$ & $\begin{array}{l}9.61 \times 10^{-3} \\
9.72 \times 10^{-3}\end{array}$ & $\begin{array}{l}7.68 \times 10^{-8} \\
4.19 \times 10^{-7}\end{array}$ & & [Ma et al., 2002] \\
\hline $\begin{array}{l}\text { murine-mAb (BIWA-1) } \\
\text { (U36) } \\
\text { chimeric-mAb (BIWA-2) } \\
\text { humanized-mAb (BIWA-4) } \\
\text { (BIWA-8) }\end{array}$ & GST/CD44v3-v10 & $\begin{array}{l}1.3 \times 10^{5} \\
1.5 \times 10^{4} \\
1.7 \times 10^{5} \\
6.5 \times 10^{4} \\
7.5 \times 10^{4}\end{array}$ & $\begin{array}{l}4.2 \times 10^{-5} \\
1.7 \times 10^{-4} \\
4.1 \times 10^{-5} \\
2.2 \times 10^{-4} \\
6.3 \times 10^{-5}\end{array}$ & $\begin{array}{l}3.2 \times 10^{-10} \\
1.1 \times 10^{-8} \\
2.4 \times 10^{-10} \\
3.4 \times 10^{-9} \\
8.4 \times 10^{-10}\end{array}$ & & [Verel et al., 2002] \\
\hline $\begin{array}{l}\text { Fc } \alpha \text { RI } \\
\text { Fc } \alpha\end{array}$ & $\begin{array}{l}\text { Fc } \alpha \\
\text { Fc } \alpha \text { RI }\end{array}$ & $\begin{array}{l}2.2 \times 10^{5}\left(k_{\mathrm{a}} 1\right) \\
1.4 \times 10^{5}\left(k_{\mathrm{a}} 2\right) \\
8.4 \times 10^{4}\left(k_{\mathrm{a}} 1\right) \\
2.2 \times 10^{4}\left(k_{\mathrm{a}} 2\right)\end{array}$ & $\begin{array}{l}3.7 \times 10^{-2}\left(k_{\mathrm{d}} 1\right) \\
6.8 \times 10^{-2}\left(k_{\mathrm{d}} 2\right) \\
1.5 \times 10^{-2}\left(k_{\mathrm{d}} 1\right) \\
2.0 \times 10^{-3}\left(k_{\mathrm{d}} 2\right)\end{array}$ & $\begin{array}{l}169 \pm 10 \times 10^{-9}\left(K_{\mathrm{D}} 1\right) \\
494 \pm 101 \times 10^{-9}\left(K_{\mathrm{D}} 2\right) \\
180 \times 10^{-9}\left(K_{\mathrm{D}} 1\right) \\
1170 \times 10^{-9}\left(K_{\mathrm{D}} 2\right)\end{array}$ & & [Herr et al., 2003] \\
\hline $\begin{aligned} \text { anti-NP Fab } & (9 \mathrm{~T} 7) \\
& (9 \mathrm{~T} 8) \\
& (9 \mathrm{~T} 10) \\
& (9 \mathrm{~T} 13)\end{aligned}$ & $\mathrm{NP}_{2}$-HEL & $\begin{array}{l}5.5 \times 10^{5} \\
5.4 \times 10^{5} \\
1.8 \times 10^{5} \\
3.9 \times 10^{5}\end{array}$ & $\begin{array}{l}1.3 \times 10^{-2} \\
7.5 \times 10^{-3} \\
2.3 \times 10^{-3} \\
2.5 \times 10^{-3}\end{array}$ & $\begin{array}{l}2.4 \times 10^{-8} \\
1.4 \times 10^{-8} \\
1.3 \times 10^{-8} \\
6.4 \times 10^{-8}\end{array}$ & & [Sagawa et al., 2003] \\
\hline $\begin{array}{l}\mathrm{mAb}(\mathrm{mAb} 11) \\
\qquad(\mathrm{mAb} 5) \\
(\mathrm{mAb} 12)\end{array}$ & bovine cardiac troponin I (cTnI) & $\begin{array}{l}1.15 \times 10^{4} \\
8.21 \times 10^{3} \\
1.40 \times 10^{3}\end{array}$ & $\begin{array}{l}5.88 \times 10^{-3} \\
5.49 \times 10^{-3} \\
6.10 \times 10^{-3}\end{array}$ & $\begin{array}{l}5.10 \times 10^{-7} \\
6.71 \times 10^{-7} \\
4.35 \times 10^{-6}\end{array}$ & $\begin{array}{l}1 \\
1 \\
1\end{array}$ & [Liu et al., 2003] \\
\hline $\begin{array}{l}\mathrm{NP}_{4.4} \text {-BSA } \\
\mathrm{NP}_{18.6} \text {-BSA } \\
\mathrm{NP}_{36.2} \text {-CGG } \\
\mathrm{NP}_{9.0} \text {-MMG } \\
\mathrm{NP}_{31.5}-\mathrm{MMG} \\
\mathrm{NP}_{56.5}-\mathrm{MMG} \\
\mathrm{NP}_{159}-\mathrm{MMG}\end{array}$ & anti-NP IgG1 (A6-hCu) & & & $\begin{array}{l}2.13 \times 10^{-8} \\
1.52 \times 10^{-9} \\
1.14 \times 10^{-9} \\
7.69 \times 10^{-9} \\
2.27 \times 10^{-9} \\
1.96 \times 10^{-9} \\
1.21 \times 10^{-9}\end{array}$ & $\begin{array}{l}0.59 \\
0.71 \\
0.40 \\
0.14 \\
0.15 \\
0.14 \\
0.16\end{array}$ & [Tobita et al., 2003] \\
\hline $\begin{array}{r}\text { hybridoma supernatant }(\mathrm{H} 3) \\
\qquad \begin{array}{r}\text { (A1) } \\
(\mathrm{B} 1) \\
(\mathrm{C} 1) \\
\text { (D1) } \\
\text { (B2) }\end{array}\end{array}$ & Fc-specific IgG & $\begin{array}{l}3.90[1]^{\mathrm{g}} \times 10^{5} \\
4.54[3] \times 10^{5} \\
3.79[3] \times 10^{5} \\
4.37[5] \times 10^{5} \\
4.12[3] \times 10^{5} \\
2.18[4] \times 10^{5}\end{array}$ & $\begin{array}{l}1.5[1]^{\mathrm{h}} \times 10^{-4} \\
2.1[1] \times 10^{-4} \\
4.0[1] \times 10^{-4} \\
4.5[1] \times 10^{-4} \\
7.5[1] \times 10^{-4} \\
2.4[1] \times 10^{-4}\end{array}$ & $\begin{array}{l}0.39[2]^{\mathrm{i}} \\
0.45[1] \\
1.06[3] \\
1.04[2] \\
1.82[2] \\
1.10[4]\end{array}$ & & [Canziani et al., 2003] \\
\hline $\begin{array}{r}\text { mouse-mAb (BBK-4) } \\
\text { humanized scFv (A12-1) } \\
\qquad \begin{aligned} \text { (D4-2) } \\
\text { (E4-1) } \\
\text { (A-E5) } \\
\text { (B-A5) } \\
\text { (B-G2) }\end{aligned}\end{array}$ & human 4-1BB receptor & $\begin{array}{l}1.25 \times 10^{3} \\
4.18 \times 10^{4} \\
1.29 \times 10^{3} \\
1.72 \times 10^{2} \\
5.07 \times 10^{2} \\
4.51 \times 10^{2} \\
2.32 \times 10^{2}\end{array}$ & $\begin{array}{l}5.88 \times 10^{-7} \\
7.62 \times 10^{-4} \\
8.35 \times 10^{-6} \\
6.42 \times 10^{-6} \\
9.39 \times 10^{-6} \\
1.07 \times 10^{-5} \\
1.16 \times 10^{-5}\end{array}$ & $\begin{array}{l}4.70 \times 10^{-10} \\
1.82 \times 10^{-8} \\
6.47 \times 10^{-9} \\
3.73 \times 10^{-8} \\
1.85 \times 10^{-8} \\
2.38 \times 10^{-8} \\
4.99 \times 10^{-8}\end{array}$ & & [Son et al., 2004] \\
\hline $\begin{array}{l}\text { a monomeric protein }(\mathrm{Ag}-1, \mathrm{MW} 47 \mathrm{kDa}) \\
\text { a monomeric polypeptide (Ag-2, MW } 9.4 \mathrm{kDa}) \\
\qquad\left(\mathrm{Ag}-2^{\prime}, \mathrm{MW} 4.1 \mathrm{kDa}\right)\end{array}$ & $\begin{array}{l}\mathrm{mAb}-1 \\
\mathrm{mAb}-2 \\
\mathrm{mAb}-2\end{array}$ & $\begin{array}{l}4.6(0.1) \times 10^{5} \\
6.6(1.5) \times 10^{5} \\
2.7(0.6) \times 10^{6}\end{array}$ & $\begin{array}{l}1.2(0.1) \times 10^{-3} \\
2.1(0.5) \times 10^{-5} \\
1.6(0.2) \times 10^{-5}\end{array}$ & $\begin{array}{l}2.5(0.5) \times 10^{-9} \\
3.3(1.1) \times 10^{-11} \\
6.2(0.8) \times 10^{-12}\end{array}$ & & [Drake et al., 2004] \\
\hline $\begin{array}{l}\text { anti-Le }{ }^{\mathrm{y}} \text { antibody (hu3S193) } \\
\text { (hu3S193-CalichDMH) }\end{array}$ & $L e^{y}-B S A$ & $\begin{array}{l}8.3 \times 10^{4} \\
3.7 \times 10^{4}\end{array}$ & $\begin{array}{l}1.0 \times 10^{-3} \\
3.3 \times 10^{-3}\end{array}$ & $\begin{array}{l}1.3 \times 10^{-8} \\
8.9 \times 10^{-8}\end{array}$ & & [Boghaert et al., 2004] \\
\hline linear peptide 12p1 (RINNIPWSEAMM) & HIV envelope glycoprotein (YU2 gp120) & & & $3.65 \times 10^{-6}$ & 1 & [Biorn et al., 2004] \\
\hline anti-mAb (X1BRCTb) & mAb (L3BRCT) & $1.31 \times 10^{4}$ & & $1.02 \times 10^{-9}$ & & [Beernink et al., 2005] \\
\hline
\end{tabular}

(Table 1) contd.... 
(Table 1) contd....

\begin{tabular}{|c|c|c|c|c|c|c|}
\hline Target a & Probe b & Ka $(M-1 s-1) c$ & $K d(s-1) d$ & $K D(M)$ e & $\mathbf{n} \mathbf{f}$ & Ref. \\
\hline human Fc $\gamma \mathrm{RI}$ (rsCD64) & $\begin{array}{l}\text { hCG-cPIPP } \\
\text { ARP7073-2F5 }\end{array}$ & $\begin{array}{l}1.7 \times 10^{6} \\
1.5 \times 10^{6}\end{array}$ & $\begin{array}{l}1.8 \times 10^{-4} \\
3.8 \times 10^{-4}\end{array}$ & $\begin{array}{l}1.06 \times 10^{-11} \\
2.53 \times 10^{-11}\end{array}$ & & [Paetz et al., 2005] \\
\hline $\begin{array}{l}\operatorname{sc}(\mathrm{Fv})_{2} \\
\operatorname{sc}(\mathrm{Fv})_{2} \text {-Ang } I\end{array}$ & $\begin{array}{l}\text { pancarcinoma tumor-associated antigen } \\
\text { (TAG-72) }\end{array}$ & & & $\begin{array}{l}8.47 \times 10^{-8} \\
1.19 \times 10^{-7}\end{array}$ & 2 & [Wittel et al., 2005] \\
\hline $\begin{array}{l}\text { HHHHHH-protein } \\
\text { HHHHHH } \\
\text { HHHHHHHHHH } \\
\text { MDGHPERHDAGDHHHHHGVRQ } \\
\text { RHDAGDHHHHHGVRQ }\end{array}$ & Fab & $\begin{array}{l}7.3 \\
9.9 \\
62.4 \\
11.8 \\
21.5\end{array}$ & $\begin{array}{l}23.82 \\
5.78 \\
6.56 \\
0.85 \\
0.82\end{array}$ & $\begin{array}{l}3.26 \times 10^{-6} \\
5.80 \times 10^{-7} \\
1.05 \times 10^{-7} \\
7.20 \times 10^{-8} \\
3.80 \times 10^{-8}\end{array}$ & & [Ja et al., 2005] \\
\hline $\begin{array}{l}\text { MDGHPERHDAGDHHHHHGVRQWRLISTG-MBP } \\
\text { NSPGRFRHHHVLARRHALYR } \\
\text { MKVRRDVMRWHHHHRMARRKANR-MBP }\end{array}$ & & $\begin{array}{l}52.5 \\
17.4 \\
40.9\end{array}$ & $\begin{array}{l}0.97 \\
0.27 \\
0.31\end{array}$ & $\begin{array}{l}1.85 \times 10^{-8} \\
1.55 \times 10^{-8} \\
7.60 \times 10^{-9}\end{array}$ & & \\
\hline $\mathrm{scFv}-\mathrm{His}_{6}$ & $\begin{array}{l}\text { Progesterone } \\
5 \beta \text {-DHP } \\
5 \alpha-\text { DHP }\end{array}$ & & & $\begin{array}{l}(0.020 \pm 0.009) \times 10^{-9} \\
(0.028 \pm 0.009) \times 10^{-9} \\
(0.097 \pm 0.028) \times 10^{-9}\end{array}$ & $\begin{array}{l}1 \\
1 \\
1\end{array}$ & [Dubreuil et al., 2005] \\
\hline interleukin-8 (IL-8) & $\begin{array}{l}\text { mAb (MAB208) } \\
\text { (JK8-2) }\end{array}$ & $\begin{array}{l}1.46 \times 10^{6} \\
6.65 \times 10^{5}\end{array}$ & $\begin{array}{l}2.06 \times 10^{-6} \\
2.31 \times 10^{-3}\end{array}$ & $\begin{array}{l}1.41 \times 10^{-12} \\
3.48 \times 10^{-9}\end{array}$ & $\begin{array}{l}1 \\
1\end{array}$ & [Yang et al., 2005] \\
\hline human Fc $\gamma \mathrm{RI}$ (rsCD64) & human IgG & $1.7 \times 10^{6}$ & $1.8 \times 10^{-4}$ & $1.1 \times 10^{-10}$ & 1 & [Paetz et al., 2005] \\
\hline $\begin{array}{r}\text { HSA }(\mathrm{pH} \mathrm{5.0)} \\
(\mathrm{pH} 5.5) \\
(\mathrm{pH} 6.0) \\
(\mathrm{pH} 6.5) \\
(\mathrm{pH} 7.0) \\
\text { shFcRn }(\mathrm{pH} 5.0) \\
(\mathrm{pH} 5.5) \\
(\mathrm{pH} 6.0) \\
(\mathrm{pH} 6.5) \\
(\mathrm{pH} \mathrm{7.0)}\end{array}$ & HSA & $\begin{array}{l}(14 \pm 2.5) \times 10^{3} \\
(9.3 \pm 0.0) \times 10^{3} \\
(7.7 \pm 1.1) \times 10^{3} \\
(1.0 \pm 0.2) \times 10^{3} \\
(0.5 \pm 0.5) \times 10^{3} \\
(35.9 \pm 4.1) \times 10^{3} \\
(27.0 \pm 2.6) \times 10^{3} \\
(16.6 \pm 1.5) \times 10^{3} \\
(5.9 \pm 1.3) \times 10^{3} \\
(2.6 \pm 1.2) \times 10^{3}\end{array}$ & $\begin{array}{l}(5.8 \pm 0.3) \times 10^{-3} \\
(6.9 \pm 0.6) \times 10^{-3} \\
(8.5 \pm 3.0) \times 10^{-3} \\
(21.0 \pm 3.7) \times 10^{-3} \\
(58.0 \pm 61) \times 10^{-3} \\
(6.3 \pm 0.7) \times 10^{-3} \\
(7.8 \pm 0.4) \times 10^{-3} \\
(15.2 \pm 0.4) \times 10^{-3} \\
(37.3 \pm 1.7) \times 10^{-3} \\
(138 \pm 13.4) \times 10^{-3}\end{array}$ & $\begin{array}{l}(0.4 \pm 0.0) \times 10^{-6} \\
(0.7 \pm 0.2) \times 10^{-6} \\
(1.1 \pm 0.5) \times 10^{-6} \\
(21.6 \pm 5.8) \times 10^{-6} \\
(116.6 \pm 63.6) \times 10^{-6} \\
(0.2 \pm 0.0) \times 10^{-6} \\
(0.3 \pm 0.0) \times 10^{-6} \\
(0.9 \pm 0.1) \times 10^{-6} \\
(6.5 \pm 1.1) \times 10^{-6} \\
(72.8 \pm 60.3) \times 10^{-6}\end{array}$ & $\begin{array}{c}1.1 \pm 0.1 \\
\\
1.1 \pm 0.1\end{array}$ & [Chaudhury et al., 2006] \\
\hline $\begin{array}{l}\text { hen egg white lysozyme (HEL) } \\
\text { ovalbumin (OVA) } \\
\text { bovine serum albumin (BSA) }\end{array}$ & $\begin{array}{r}\text { anti-HEL mAb (HC1) } \\
(\mathrm{HC} 2) \\
\text { anti-OVA mAb (O1-1) } \\
(\text { YOVA) } \\
\text { anti-BSA mAb (KM10) }\end{array}$ & $\begin{array}{l}1.0 \times 10^{8} \\
1.5 \times 10^{8} \\
1.6 \times 10^{4} \\
3.9 \times 10^{4} \\
1.5 \times 10^{5}\end{array}$ & $\begin{array}{l}3.1 \times 10^{-3} \\
2.7 \times 10^{-3} \\
7.7 \times 10^{-2} \\
3.5 \times 10^{-4} \\
4.5 \times 10^{-4}\end{array}$ & $\begin{array}{l}3.10 \times 10^{-11} \\
1.82 \times 10^{-11} \\
4.81 \times 10^{-6} \\
8.97 \times 10^{-9} \\
3.00 \times 10^{-9}\end{array}$ & $\begin{array}{l}1.8 \\
2.1 \\
0.9 \\
1.0 \\
1.1\end{array}$ & [Oda et al., 2006] \\
\hline prostate-specific antigen (PSA) & anti-PSA mAb & $(4.1 \pm 0.6) \times 10^{4}$ & $(4.5 \pm 0.6) \times 10^{-5}$ & $(1.1 \pm 0.2) \times 10^{-9}$ & 1 & [Katsamba et al., 2006] \\
\hline $\mathrm{scFv}$ & $\begin{array}{l}\text { Lewis } x\left(\operatorname{Le}^{x}\right) \\
\text { sialyl } \operatorname{Le}^{x}\left(\operatorname{sLe}^{x}\right)\end{array}$ & & & $\begin{array}{l}(3.5 \pm 0.7) \times 10^{-5} \\
(2.6 \pm 0.7) \times 10^{-5}\end{array}$ & $\begin{array}{l}1 \\
1\end{array}$ & [Johansson et al., 2006] \\
\hline $\begin{array}{l}\operatorname{scFv} \\
\text { covalent diabody }\end{array}$ & murine laminin-1 & & & $\begin{array}{l}9.9 \times 10^{-8} \\
4.4 \times 10^{-10}\end{array}$ & $\begin{array}{l}1 \\
2\end{array}$ & [Huang et al., 2006] \\
\hline
\end{tabular}

where $\mathrm{d} R / \mathrm{d} t$ is the rate of change of the SPR response signal, $R$ and $R_{\max }$ are the measured and maximum response signal measured with binding, $C$ is the injected concentration (M) of the $\mathrm{Ag}, k_{\mathrm{a}}$ is the association rate constant or on-rate $\left(\mathrm{M}^{-1} \mathrm{~s}^{-1}\right)$ and $k_{\mathrm{d}}$ is the dissociation rate or off-rate $\left(\mathrm{s}^{-1}\right)$ (Fig. 3A). The binding constant, i.e., association constants $K_{\mathrm{A}}$, may be calculated as $K_{\mathrm{A}}=k_{\mathrm{a}} / k_{\mathrm{d}}\left(\mathrm{M}^{-1}\right)$. At equilibrium, $\mathrm{d} R / \mathrm{d} t=0$ and Eq. [3] can be rewritten as:

$$
R_{\mathrm{eq}} / C=K_{\mathrm{A}} R_{\max }-K_{\mathrm{A}} R_{\mathrm{eq}}
$$

Therefore, the steady state association constant $K_{\mathrm{A}}$ can be obtained from a plot of $R_{\text {eq }} / C$ versus $R_{\text {eq }}$ and the dissociation constant $K_{\mathrm{D}}$ can be calculated as $1 / K_{\mathrm{A}}$ (Fig. 3B).

\subsection{Association Kinetics Analysis}

Equation [3] may be rearranged as:

$$
\mathrm{d} R / \mathrm{dt}=k_{\mathrm{a}} C R_{\max }-\left(k_{\mathrm{a}} C+k_{\mathrm{d}}\right) R
$$

Thus a plot of $\mathrm{d} R / \mathrm{dt}$ against will theoretically be a straight line with slope $-\left(k_{\mathrm{a}} C+k_{\mathrm{d}}\right)$ for interaction-controlled kinetics. The initial binding rate (at $\mathrm{R}=0$ ) is directly proportional to the analyte concentration and can be used for concentration measurements. If $R_{\max }$ is known, both $k_{\mathrm{a}}$ and $k_{\mathrm{d}}$ can be determined from a single association sensorgram. $R_{\max }$ is however often difficult to determine experimentally, since a high analyte concentration is required to fully saturate the surface. A preferable approach is to measure the association sensorgram at several different analyte concentrations. For analysis of the on and off rates, a plot of the change in total detector response $\left(\mathrm{d} R / \mathrm{d}_{t}\right)$ versus $R$ gives a value $S$ as the slope which relates the on and off rates as follows:

$$
S=k_{\mathrm{a}} C+k_{\mathrm{d}}
$$

A plot of $S$ against $C$ will be a straight line with slope $k_{\mathrm{a}}$ (Fig. 3C). In theory, the intercept on the ordinate $(C=0)$ give $k_{\mathrm{d}}$ : in practice, however, this cannot be used as a reliable measure of the dissociation rate constant if $k_{\mathrm{a}} C>k_{\mathrm{d}}$. A more accurate way to obtain this value is by direct measurement of the dissociation from saturated binding sites into a buffer solution flow that contains no $\mathrm{Ag}$ and the dissociation is quantified by:

$$
\ln \left(R_{\mathrm{o}} / R_{\mathrm{t}}\right)=k_{\mathrm{d}}\left(\mathrm{t}-\mathrm{t}_{\mathrm{o}}\right)
$$

where $R_{\mathrm{o}}$ is the initial response level at $\mathrm{t}_{\mathrm{o}}$ and $R$ and tepresent values obtained along the dissociation curve. 


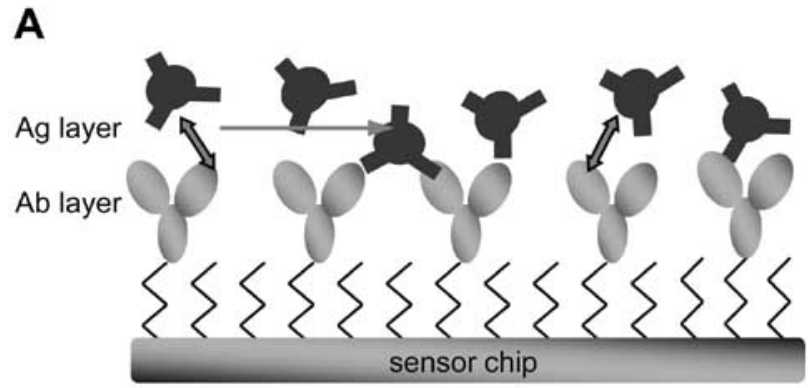

B

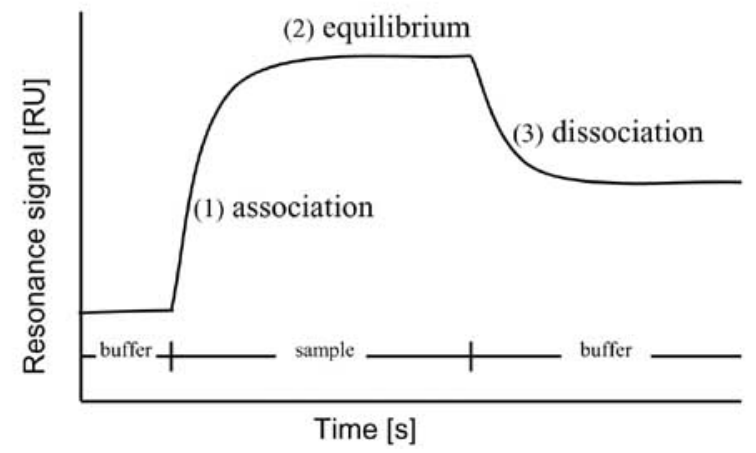

Fig. (2). (A) Schematic diagram depicting the interaction of $\mathrm{Ag}$ with $\mathrm{Ab}$ at liquid-solid interface. (B) Schematic sensorgram, showing association, equilibrium and dissociation phases. When $\mathrm{Ag}$ is injected in a discrete pulse across an $\mathrm{Ab}$ surface, the resulting sensorgram can be divided into three essential phases: (1) Association of $\mathrm{Ag}$ with $\mathrm{Ab}$ during sample injection; (2) Equilibrium or steady state during sample injection, where the rate of $\mathrm{Ag}$ binding is balanced by dissociation from the complex; (3) Dissociation of $\mathrm{Ag}$ from the surface during buffer flow. RU represents response unit.

\section{ONE TO ONE INTERACTION MODE}

\subsection{Data Analysis}

The theory of kinetic measurements for an $\mathrm{Ag}-\mathrm{Ab}$ reaction analyzed in terms of the one to one interaction model has been described by Oda and Azuma (2000). The association rate constant, $k_{\mathrm{a}}$, can be determined by carrying out a series of interaction experiments with different $\mathrm{Ag}$ concentration $(C)$ and by measuring the binding rate, $\mathrm{d} R / \mathrm{d} t$, as a function of the SPR response $(R)$ (Eq. [5]). Using linear transformation of the primary data, plots of $\mathrm{d} R / \mathrm{d} t$ versus $\mathrm{R}$ are made for a range of $\mathrm{Ag}$ concentrations and the slopes of these curves, $S=k_{\mathrm{a}} C+k_{\mathrm{d}}$ (Eq. [6]), are determined. The $S$ values are then plotted versus concentrations, and the new line obtained will have a slope corresponding to $k_{\mathrm{a}}$. The dissociation rate constant, $k_{\mathrm{d}}$, can be calculated from the dissociation phase described by Eq. [7]. A plot of $\ln \left(R_{\mathrm{o}} / R_{\mathrm{t}}\right)$ versus $t-t_{\mathrm{o}}$ gives a straight line with slope $k_{\mathrm{d}}$. The apparent binding (or association) constant, $K_{\mathrm{A}}$, for the Ag-Ab interaction can then be determined as the ratio $K_{\mathrm{A}}=k_{\mathrm{a}} / k_{\mathrm{d}}\left(\right.$ or dissociation constant $\left.K_{\mathrm{D}}=k_{\mathrm{d}} / k_{\mathrm{a}}\right)$.

\subsection{Examples}

\subsubsection{Monovalent scfv-Monovalent Haptenic Ag Interaction}

Dubreuil and co-workers (2005) investigated the binding of wild-type anti-progesterone (i) $\mathrm{scFv}_{\mathrm{C} 12 \mathrm{G} 11}$, (ii) mutant $\mathrm{B} 14$, and (iii) mutant $\mathrm{B} 14+\mathrm{Lys}^{\mathrm{H} 31}-\mathrm{His}^{\mathrm{H} 32}$ to soluble and unmodified progesterone, $5 \alpha$ - and $5 \beta$-dihydroprogesterone (DHP), respectively, by competition SPR experiments to develop a sensitive and highly specific in vitro immunoassay. The results obtained showed that mutant B14
+ Lys $^{\mathrm{H} 31}$-His ${ }^{\mathrm{H} 32}$ is much more specific than the wild-type antibody $\mathrm{scFv}$ fragment with deduced $K_{\mathrm{D}}$ values in the nanomolar range (5.3 and $2.4 \times 10^{-9} \mathrm{M}$ for $5 \alpha$-DHP and $5 \beta$-DHP, respectively) versus 97 $\mathrm{x} 10^{-12} \mathrm{M}\left(5 \alpha\right.$-DHP) and $28 \times 10^{-12} \mathrm{M}(5 \beta$-DHP) for the wild-type $\mathrm{scFv}$ fragment, whereas the affinity for progesterone remains in the picomolar range. Moreover, the improvement in specificity for their best mutant $\mathrm{B} 14+\mathrm{Lys}^{\mathrm{H} 31}$-His ${ }^{\mathrm{H} 32}$ compared with the wild-type $\mathrm{scFv}_{\mathrm{C} 12 \mathrm{G} 11}$ was calculated from measurement obtained under identical experimental conditions. It showed an increase in discrimination against $5 \alpha$-DHP and 5 $\beta$-DHP, 23-fold and 15-fold, respectively, compared with the starting $\mathrm{scFv}_{\mathrm{C} 12 \mathrm{G} 11}$ antibody. These improvements were calculated as cross-reaction percentages from experimentally measured $K_{\mathrm{D}}$ values. Furthermore, the lower crossreactivity of mutant $\mathrm{B} 14+\mathrm{Lys}^{\mathrm{H} 31}-\mathrm{His}^{\mathrm{H} 32}$ was associated with a moderate 3.8- fold increase in the dissociation constant for progesterone $\left(75 \times 10^{-12} \mathrm{M}\right.$ versus $\left.20 \times 10^{-12} \mathrm{M}\right)$.

A

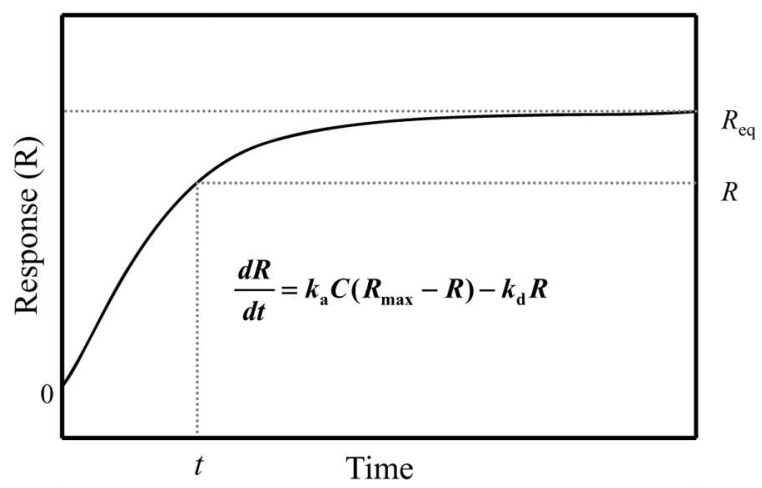

B

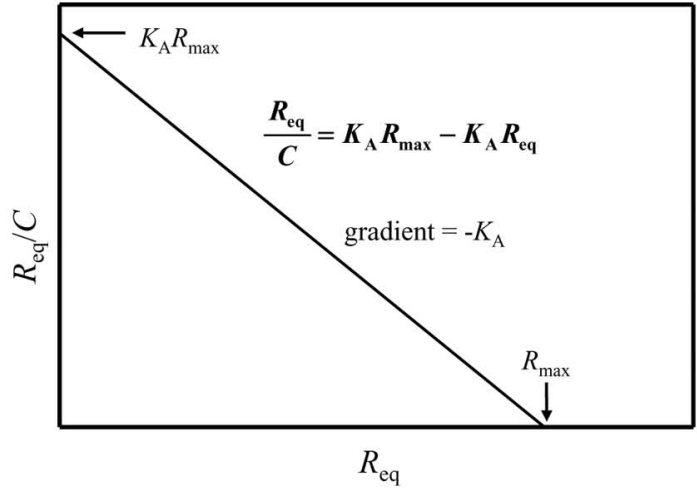

C

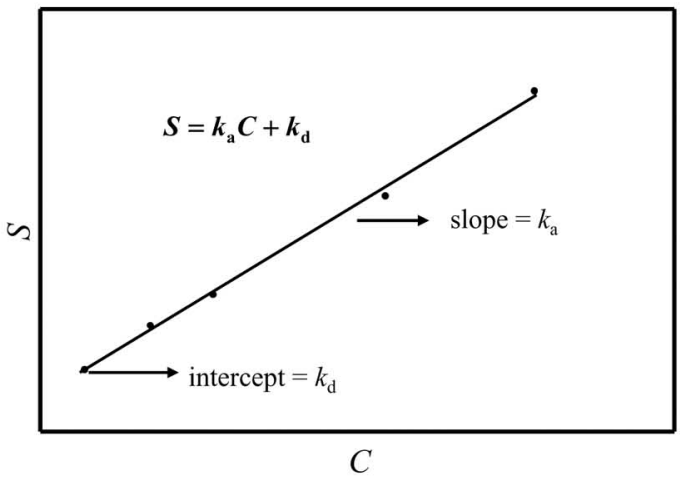

Fig. (3). (A) Simulated sensorgram illustrating affinity and kinetic measurement of $\mathrm{Ag}-\mathrm{Ab}$ interaction according to Eq. [3]. (B) A plot of $R_{\mathrm{eq}} / C$ against $R_{\text {eq }}$ at different $\mathrm{Ag}$ concentrations according to Eq. [4] thus gives a straight line from which $R_{\max }$ and $K_{\mathrm{A}}$ can be calculated. (C) A plot of slope value $S$ versus $\mathrm{Ag}$ concentration $C$ according to Eq. [6]. 


\subsubsection{Monovalent scfv-Monomeric Protein Ag Interaction}

Son and co-workers (2004) determined the kinetic constants for an interaction between human $4-1 \mathrm{BB}$ and soluble humanized $\mathrm{scFv}$ antibodies by SPR measurements. In this work, the humanization of agonistic anti-human 4-1BB mAb, BBK-4, using a phage display library, was presented. They prepared the combinatorial library by incorporating murine and human alternative at positions representing buried residues that might affect the structural integrity of the antigen binding site. Six humanized single chain $\mathrm{Fv}(\mathrm{scFv}$ ) fragments were selected from the combinatorial library expressing phage-displayed humanized $\mathrm{scFv}$ and found to retain the epitope specificity of the original mAb but had affinities of lower than $1 / 10$ of the original. To obtain an association rate constant $\left(k_{\mathrm{a}}\right)$, the binding rate at five different humanized $\mathrm{scFv}$ concentrations ranging from 5 to $40 \mu \mathrm{g} / \mathrm{ml}$ in PBS was determined using SPR. Dissociation rate constants $\left(k_{\mathrm{d}}\right)$ were the average of five measurements obtained by analyzing the dissociation phase at an increased flow rate $(40 \mu \mathrm{l} / \mathrm{min})$. The dissociation constant $\left(K_{\mathrm{D}}\right)$ calculated from $K_{\mathrm{D}}=k_{\mathrm{d}} / k_{\mathrm{a}}$ and was found to be $(0.65 \sim 4.99) \times$ $10^{-8}$. These results suggest that humanized anti-human 4-1BB scFvs can be used as a valuable reagent for clinical application.

\subsubsection{Monovalent Fab-Monovalent Haptenic Ag Interaction}

Canziani et al., (2004) determined kinetic parameters by SPR using the BIAcore biosensor system for the $\mathrm{Ag}-\mathrm{Ab}$ interaction using a group of anti-(4-hydroxy-3-nitrophenyl)acetyl (NP) mAb whose differences in amino acid sequences had arisen only from somatic hypermutation. To investigate monovalent interaction, they prepared $\mathrm{F}_{\mathrm{ab}}$ fragments of 9TX mAbs and passed them over the surface of the BIAcore SPR sensor chip, on which the NP-protein conjugates was immobilized. The kinetics of the binding of these $\mathrm{F}_{\mathrm{ab}} \mathrm{s}$ to the immobilized NP-hen egg white lysozyme (HEL) were essentially the same as to the immobilized NP-bovine serum albu$\min$ (BSA). The validity of the estimation of $k_{\mathrm{a}}\left(5.5 \times 10^{5}\right.$ for 9T7Fab, $5.4 \times 10^{5}$ for 9T8, Fab $1.8 \times 10^{5}$ for 9T10 Fab, $3.9 \times 10^{5}$ for $9 \mathrm{~T} 13 \mathrm{Fab})$ or $k_{\mathrm{b}}\left(1.3 \times 10^{-2}\right.$ for 9T7Fab, $7.5 \times 10^{-3}$ for $9 \mathrm{~T} 8$, Fab $2.3 \times 10^{-3}$ for 9T10 Fab, $2.5 \times 10^{-3}$ for 9T13 Fab) was confirmed by the fact that the $K_{\mathrm{A}}$ values calculated from the two rate constants were similar to the $K_{\mathrm{A} \text {,eq }}$ determined from Scatchard analysis. The kinetic analysis revealed that dissociation rates decreased during evolution. In addition, the $k_{\mathrm{a}}$ values of 9T7/9T8 were larger than those of 9T10/9T13. These results suggested that the mechanism for the $\mathrm{Ag}-\mathrm{Ab}$ interaction shifted from a "zipper" type to a "lockand-key" type during Ab evolution.

\subsubsection{Monovalent Fab - Monovalent Peptide Ag Interaction}

Ja and co-workers (2005) reported the interactions of antipolyhistidine Fab with various peptides from the fragment selection synthesized or expressed for kinetic analysis by SPR technique. Absolute and relative binding constants obtained from $\mathrm{mAb}$ interactions with immobilized Ags are unreliable owing to the effects of rebinding and bivalency. To avoid these problems, Fab was prepared from anti-polyhistidine $\mathrm{mAb}$ and used as the analyte. Kinetic parameters were determined using a simple 1:1 bimolecular interaction model. The assayed peptides were categorized by their dissociation rates from the $\mathrm{Fab}$, and the cited epitope, $\mathrm{His}_{6}$, bound weakest to the $\mathrm{Fab}$; the $\mathrm{His}_{6}$ peptide and the $\mathrm{His}_{6}$-tagged protein used in the original selection exhibited $K_{\mathrm{D}}$ values of 0.6 and $3 \times 10^{-6} \mathrm{M}$, respectively. Additional His residues ( $\mathrm{His}_{10}$ peptide) increased the association rate 6-fold without changing the dissociation rate significantly. In addition, kinetics measurements made by SPR, using purified Fab (antigen-binding fragment) to prevent avidity effects, demonstrated that the selected peptides bind with 10- to 75-fold higher affinities $\left(K_{\mathrm{D}}<75 \times 10^{-9} \mathrm{M}\right)$ than a hexahistidine peptide. The fragment library construction methodology described by the authors was applicable to the development of high-complexity protein or cDNA expression libraries for the identification of proteinprotein interaction domains.

\section{NONEQUIVALENT TWO-SITE INTERACTION MODE}

\subsection{Data Analysis}

The SPR response $(\mathrm{R})$ in the steady-state region is proportional to the amount of bound $\mathrm{Ag}$. The maximum response per bound $\mathrm{Ag}$ $\left(R_{\max }\right)$ in the steady-state region can be predicted by Eq [8]:

$$
R_{\max }=R_{\mathrm{Ab}} \times(\mathrm{MW} \mathrm{Ag} / \mathrm{MW} \mathrm{Ab}) \times \mathrm{RI}
$$

where $R_{\mathrm{Ab}}$ is the amount of surface immobilized $\mathrm{Ab}$ in RUs, the second term on the right side of Eq [8] is the ratio of molecular weights of the $\mathrm{Ag}$ and $\mathrm{Ab}$, and $\mathrm{RI}$ is the refractive index gradient ratio for the $\mathrm{Ag}$ and $\mathrm{Ab}$. The RI values can be measured by using differential refractometry method (Mazur et al., 2000).

Steady-state binding analysis is performed with multiple injections of different Ag concentrations over the immobilized Ab surface. Steady-state $R$ values can be converted to $r$, where $r=R /$ $R_{\max }$, and the results can then be fit by nonlinear least squares methods to a two-site equilibrium model Eq. [9]:

$$
r=\left(K_{1} C+2 K_{1} K_{2} C^{2}\right) /\left(1+K_{1} C+K_{1} K_{2} C^{2}\right)
$$

where $r$ represents the moles of bound $\mathrm{Ag}$ per mole of Ab molecule, $C$ is the unbound $\mathrm{Ag}$ concentration in equilibrium with the $\mathrm{AgAb}$ complex and is fixed by the concentration in the flow solution. $K_{1}$ and $K_{2}$ values are the macroscopic equilibrium constants for binding to the two sites.

\subsection{Examples}

Herr and co-workers (2003) investigated the binding mechanism for the interaction between a FcaRI, the receptor specific for the $\mathrm{Fc}$ region of $\operatorname{IgA}$ and a single Fc $\alpha$ homodimer by SPR experiments. Binding was monitored using both kinetic and equilibriumbased analysis. It was found that the kinetic data may be described by a bivalent ligand model in which two FcoRI molecules bind per dimeric Fc $\alpha$ molecule (Fig. 4), consistent with the 2:1 stoichiometry observed by equilibrium gel-filtration analysis. The bivalent ligand model used to analyze the data included two sequential binding events, yielding two sets of apparent on-rate and off-rate constants $\left(k_{\mathrm{a}} 1=1.7 \times 10^{5} \mathrm{M}^{-1} \mathrm{~s}^{-1}, k_{\mathrm{d}} 1=0.030 \mathrm{~s}^{-1}, k_{\mathrm{a}} 2=1.0 \times 10^{5} \mathrm{M}^{-1} \mathrm{~s}^{-1}\right.$, $\left.k_{\mathrm{d}} 2=0.044 \mathrm{~s}^{-1}\right)$. The derived or apparent rate constant for binding of the first molecule is equal to twice the intrinsic on-rate constant $\left(k_{\mathrm{a}, \mathrm{app}} 1=2 k_{\mathrm{a}} 1\right)$; and the apparent rate constant for dissociation of the second molecule is equal to twice the intrinsic off-rate con$\operatorname{stant}\left(k_{\mathrm{d}, \mathrm{app}} 2=2 k_{\mathrm{d}} 2\right)$. The intrinsic dissociation constants for binding of the first and second Fc $\alpha$ RI molecule to Fc $\alpha$ were found to be similar $\left(K_{\mathrm{D}} 1=176 \mathrm{nM} ; K_{\mathrm{D}} 2=433 \mathrm{nM}\right)($ Fig. 4A), suggesting that receptor binding to one side of Fca did not strongly influence the affinity of the second binding site, although there may be slight negative cooperativity. Comparable results were obtained by an equilibrium-based analysis of the binding data in which the data were globally fit to both one-site and two-site (bivalent ligand) models (Fig. 4B).

\section{MULTIPLE EQUIVALENT-SITE INTERACTION MODE}

\subsection{Data Analysis}

When the observed response $R$ values at high concentrations are greater than $R_{\max }$, it points to more than one binding site in these Abs. The number of binding sites may be determined from Scatchard plots of $R / C$ vs $R$ plot (Eq. [4]) by a linear regression analysis. The $R_{\max }$ value is required to convert the observed response $(R)$ to the standard binding parameter $r$ (moles of $\mathrm{Ag}$ bound/moles of probe $\mathrm{Ab}$ ), where $r=R / R_{\max }$. To obtain the binding constants, the results from the steady-state region are fitted with a multiple equivalent-site model, and Kaleidagraph is used for nonlinear least-squares optimization of the binding parameters (Carrasco et al., 2002; Teulade-Fichou et al., 2003) as follows:

$$
r=n \mathrm{~K}_{A} C /\left(1+K_{A} C\right)
$$

where $K_{A}$, the microscopic association constant, is one variable to fit; $r$ represents the moles of bound $\mathrm{Ag}$ per mole of probe $\mathrm{Ab} ; C$ is 

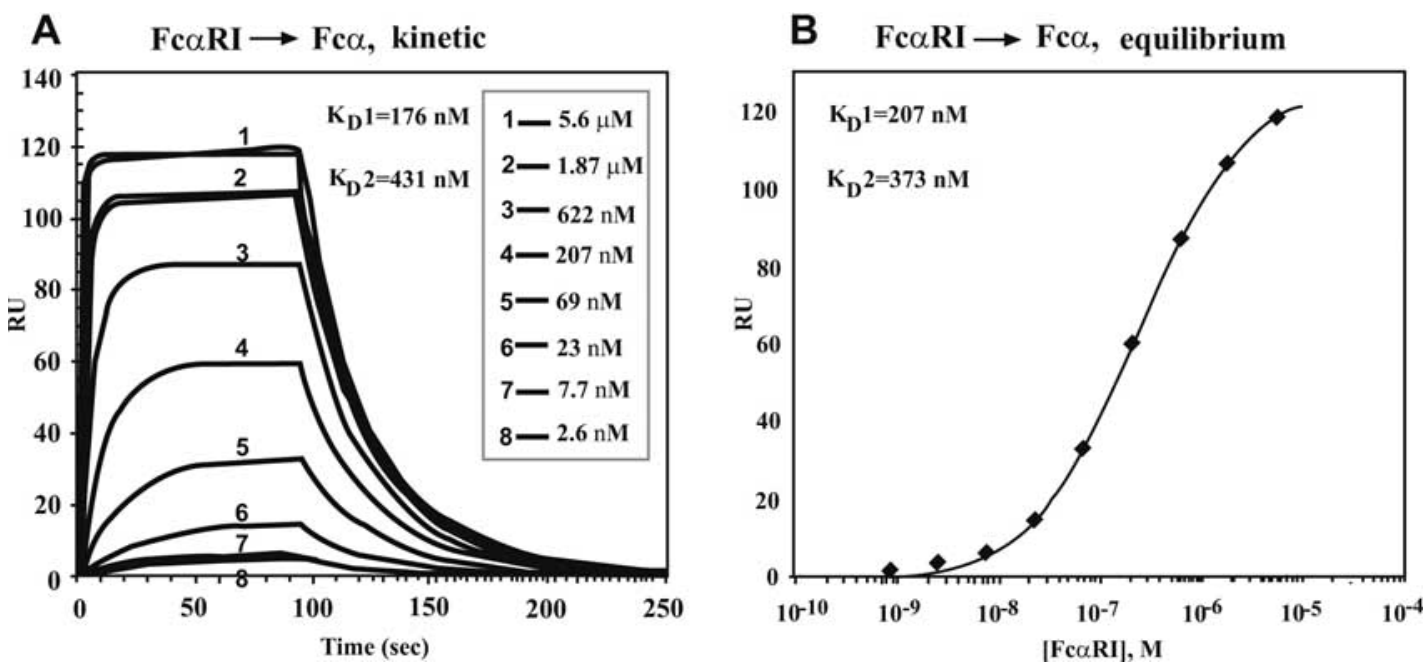

Fig. (4). Surface plasmon resonance analysis of the FcaRI:Fc $\alpha$ interaction. (A) Representative sensorgrams (colored lines) derived from injection of different concentrations of Fc $\alpha$ RI over oriented coupled Fc $\alpha$. Kinetic data were fitted globally using a bivalent ligand model (black lines). (B) Plot of equilibrium binding response $\left(R_{\mathrm{eq}}\right)$ versus concentration of injected analyte for the sensorgrams shown in $(\mathrm{A}) . K_{\mathrm{D}}$ was derived by global fitting of data from four experiments to a two-site binding model. A single representative curve is shown. Adapted with permission from Herr et al. (2003).

the concentration of the $\mathrm{Ag}$ in equilibrium with the complex and is determined by the concentration in the flow solution; $n$ is the number of equivalent $\mathrm{Ag}$-binding sites on the $\mathrm{Ab}$ and is an integer larger than one (Fig. 5A).

Equation [10] can be rearranged as follows:

$$
r / C=K_{\mathrm{A}} n-K_{\mathrm{A}} r
$$

Scatchard binding plot ( $r / C$ vs $r$ ) according to Eq. [11] is also usually used to determine the binding constant $K_{\mathrm{A}}$ and the number $n$ of equivalent binding sites on the $\mathrm{Ab}$ (Fig. 5B).
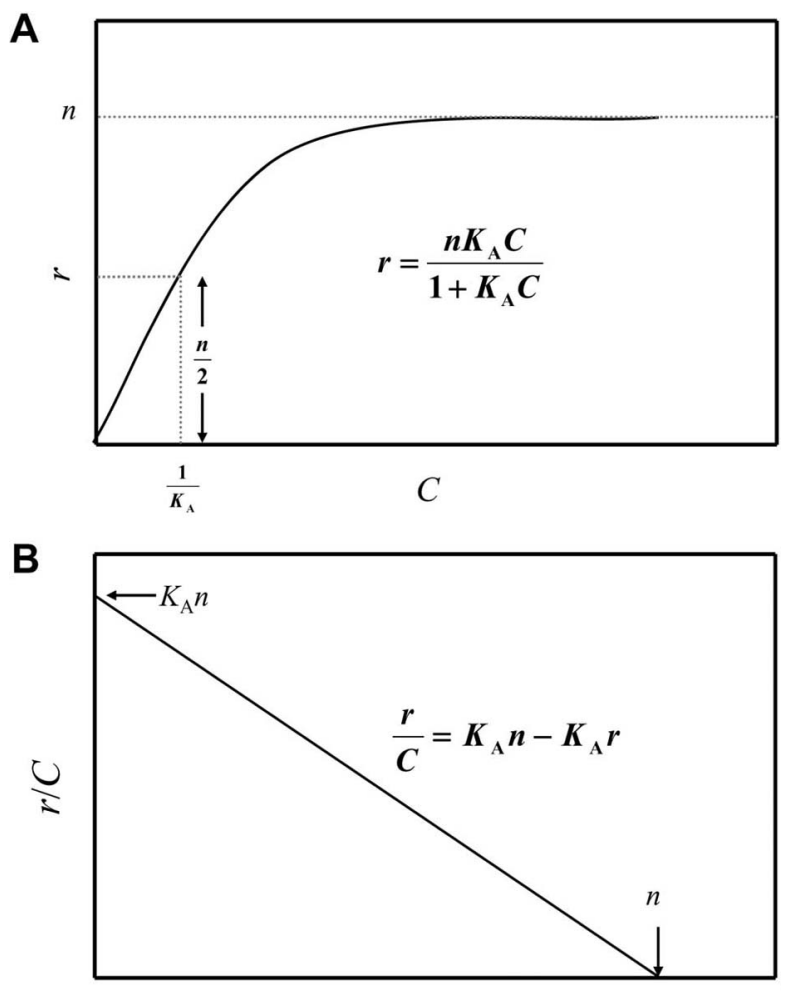

Fig. (5). (A) A binding curve of the number $r$ of equivalent binding sites on the $\mathrm{Ab}$ versus $\mathrm{Ag}$ concentration $C$ according to Eq. [10]. (B) Scatchard binding plot ( $r / C$ vs $r)$ according to Eq. [11] thus gives a straight line from which $n$ and $K_{\mathrm{A}}$ can be calculated.

\subsection{Examples}

\subsubsection{Divalent scfv-Monomeric Protein Ag Interaction}

Wittel and co-workers (2005) reported the interaction of pancarcinoma tumor-associated antigen 72 (TAG-72) with its antibody, a divalent single-chain $\mathrm{Fv}$ angiotensin II fusion construct of the monoclonal antibody $\left(\mathrm{sc}(\mathrm{Fv})_{2}\right.$-AngII). They engineered a dimeric $\mathrm{sc}(\mathrm{Fv})_{2}$-AngII fusion construct that combines the superior kinetics of covalent dimeric scFvs $\left[\mathrm{sc}(\mathrm{Fv})_{2}\right]$, recognizing the TAG-72, with the advantageous intrinsic activity of AngII. SPR studies were performed to assess the off rates as well as the real-time binding kinetics of both constructs. As shown in Fig. 6, the profiles obtained in the BIAcore evaluation of both constructs were similar. The on rates $\left(\mathrm{k}_{\text {on }}\right)$ were unaltered by the changes in the $\mathrm{sc}(\mathrm{Fv})_{2}$-AngII molecule and were determined to be $2.97 \times 10^{4}$ and $2.84 \times 10^{4}(\mathrm{Ms})$ 1 for $\mathrm{sc}(\mathrm{Fv})_{2}$-AngII and $\mathrm{sc}(\mathrm{Fv})_{2}$, respectively. Furthermore, the modifications of the $\operatorname{sc}(\mathrm{Fv})_{2}$ did not lead to an alteration of the off rate $\left(\mathrm{k}_{\text {off }}\right)$, the rate an antibody diffuses off from its target molecule. The individual values obtained for the off rates were $2.52 \times 10^{-3} \mathrm{~s}^{-1}$ for the $\mathrm{sc}(\mathrm{Fv})_{2}$-AngII and $3.38 \times 10^{-3} \mathrm{~s}^{-1}$ for the $\mathrm{sc}(\mathrm{Fv})_{2}$. Consequently, the calculation of the kinetic association constants $K_{\mathrm{A}}$ did

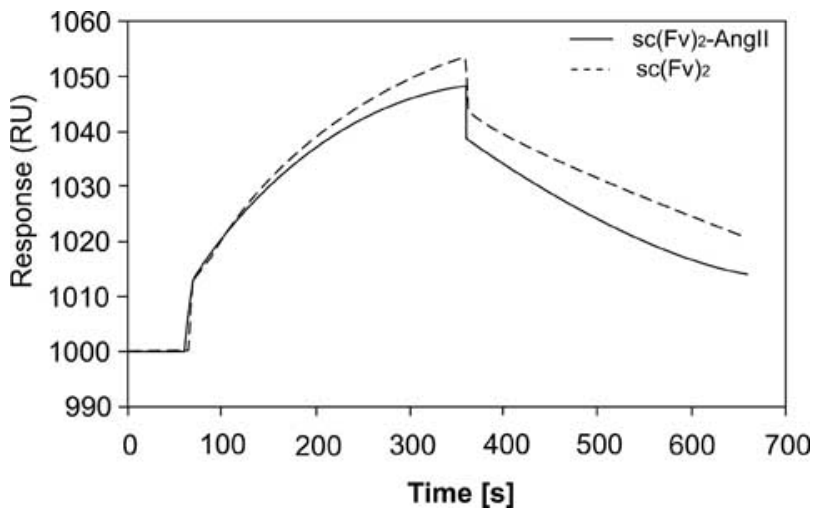

Fig. (6). Real-time binding kinetics of $\mathrm{sc}(\mathrm{Fv})_{2}$ and $\mathrm{sc}(\mathrm{Fv})_{2}$-AngII. The binding kinetics were analyzed by surface plasmon resonance to a bovine submaxillary gland mucin-coated chip. The two constructs exhibited similar on and off rates, and thus had comparable association constants, indicating that the altered design of $\operatorname{sc}(\mathrm{Fv})_{2}$ had no effect on the inherent specific binding of the $\mathrm{sc}(\mathrm{Fv})_{2}$. Reprinted with permission from Wittel et al. (2005). 
not reveal differences in the binding affinities of these two constructs and led to values of $1.18 \times 10^{7} \mathrm{M}^{-1}$ for $\mathrm{sc}(\mathrm{Fv})_{2}$-AngII and $8.42 \times 10^{6} \mathrm{M}^{-1}$ for $\mathrm{sc}(\mathrm{Fv})_{2}$, indicating that the altered design of $\mathrm{sc}(\mathrm{Fv})_{2}$ had no effect on the inherent specific binding of the $\mathrm{sc}(\mathrm{Fv})_{2}$.

\subsubsection{Divalent $\mathbf{m} A \boldsymbol{b}$ - Monovalent Haptenic Ag Interaction}

Gomes and Andreu (2002) investigated the direct kinetic analysis of small antigenic peptides, foot-and-mouth disease viral (FMDV) peptides, interacting with immobilized anti-FMDV neutralizing $\mathrm{mAb}$ using SPR protocol. High peptide concentrations up to $2.5 \mu \mathrm{M}$ and medium mAb surface densities about $1.5 \mathrm{ng} / \mathrm{mm}^{2}$ were employed to ensure measurable binding levels, and fast buffer flow rates $60 \mu \mathrm{l} / \mathrm{min}$ were used to minimize diffusion-controlled kinetics. Good reproducibility levels in the kinetic constants were obtained under these analysis conditions (standard deviations below $10 \%$ of the mean values). The local fitting was performed using the separate $k_{\mathrm{a}} / k_{\mathrm{d}}$ model and the locally fitted apparent rate constant, $k_{\mathrm{s}}$, was plotted against peptide to check the linearity $\left(k_{\mathrm{s}}=k_{\mathrm{a}} \times C+k_{\mathrm{d}}\right)$. The locally fitted response at equilibrium, $R_{\mathrm{eq}}$, was plotted against peptide concentration so that the affinity constant $K_{\mathrm{D}}$ values withdrawn from this plot could be calculated by the $k_{\mathrm{a}} / k_{\mathrm{d}}$ ratio. Application of this protocol to determine the antigenic ranking of viral peptides showed an excellent agreement between SPR and previous competition enzyme-linked immunosorbent assays ELISA on the same peptide/antibody systems.

\subsubsection{Divalent Mab-Monomeric Protein Ag Interaction}

Yang and co-workers (2005) employed SPR biosensor technique to establish the first immunoassay for the quantification of picomolar interleukin-8 (IL-8) concentrations in human saliva in a microfluidic channel. They used a sandwich assay using two monoclonal antibodies (MAB208 and JK8-2), which recognize different epitopes on the antigen (IL-8). In order to measure picomolar concentrations of salivary IL-8, the kinetic parameters, dissociation constant $\left(K_{\mathrm{D}}\right)$, of the capture antibodies (MAB208 and JK8-2) were determined using SPR. A equivalent-site binding model was used that corrected for both linear baseline drift and bulk refractive index changes. The association rate constant $\left(k_{\mathrm{a}}\right)$ and dissociation rate constant $\left(k_{\mathrm{d}}\right)$ of MAB208 were $1.46 \times 10^{6} \mathrm{M}^{-1} \mathrm{~s}^{-1}$ and $2.06 \times 10^{-6} \mathrm{~s}^{-1}$. The $k_{\mathrm{a}}$ and $k_{\mathrm{d}}$ of JK8-2 were $6.65 \times 10^{5} \mathrm{M}^{-1} \mathrm{~s}^{-1}$ and $2.31 \times 10^{-3} \mathrm{~s}^{-1}$. The dissociation constants $\left(K_{\mathrm{D}}\right)$ of MAB208 and JK8-2 were 1.41 $\mathrm{pM}$ and $3.48 \mathrm{nM}$, respectively. The $\mathrm{x} 2$ value is a standard statistical measure of the closeness of fit. These kinetic measurements revealed that MAB208 had a higher affinity for IL-8 as well as a lower dissociation rate than JK8-2. Finallly, MAB208 was chosen as the primary capture (surface) antibody, and JK8-2 was chosen as the enhancing sandwich antibody for the quantification of picomolar concentrations of IL-8 in the saliva of healthy individuals and patients with oropharyngeal squamous cell carcinoma.

\section{MULTISITE INTERACTION MODE}

\subsection{Data Analysis}

The sensorgrams (resonance units, RU, vs time) are collected with the various concentration of samples injected. The RU values are averaged over a period of time span in the steady state response region and are converted to $r$ (moles of $\mathrm{Ag}$ bound per mole of probe $\mathrm{Ab}$ ) for binding analysis as previously described in section 4.1 . The binding constants of the $\mathrm{Ag}$ to $\mathrm{Ab}$ are obtained from fitting a plot of $r$ vs Ag concentration $C$. The plot is fitted with a one-, two-, three-, or four-site interaction model (for one-site model, $K_{2}=K_{3}=K_{4}=0$; for two-site model, $K_{3}=K_{4}=0$; for three-site model, $K_{4}=0$ ) (Nguyen et al., 2002):

$r=\left(K_{1} C+2 K_{1} K_{2} C^{2}+3 K_{1} K_{2} K_{3} C^{3}+4 K_{1} K_{2} K_{3} K_{4} C^{4}\right) /\left(1+K_{1}\right.$ $\left.C+K_{1} K_{2} C^{2}+K_{1} K_{2} K_{3} C^{3}+K_{1} K_{2} K_{3} K_{4} C^{4}\right)$

\subsection{Examples}

Tobita and co-workers (2004) prepared IgG and IgM with identical combining sites to a hapten, (4-hydroxy-3-nitrophenyl) acetic acid (NP), and used SPR biosensor to evaluate the association constants $(\mathrm{Ka})$ in interactions of these Abs with Ags which differed in the size of carriers and NP valence as well as in the stoichiometry of $\mathrm{Ag}$ to $\mathrm{Ab}$ in the immune complexes. It was found that $\operatorname{IgM}$, a pentamer made up of five four-chain subunits and one J-chain, was unable to form an $\mathrm{Ag}_{1} \mathrm{Ab}_{1}$ complex with the highly haptenated $\mathrm{Ag}$, NP18.6-BSA, such that one NP18.6-BSA molecule was held by multiple contacts with Fab arms from five subunits, although IgM was capable of forming an $\mathrm{Ag}_{4} \mathrm{Ab}_{1}$ complex in which each subunit was bound to one NP18.6-BSA molecule. It was also found that IgM was superior to IgG in interactions with large Ags of low hapten density. The $K$ a values of IgM to these Ags were estimated to be $\sim 1 \times 10^{9} \mathrm{M}^{-1}$, about 20-fold higher than those of IgG. Reduction of inter-subunit and inter-chain disulfide bonds resulted in a decrease in $\mathrm{Ka}$ values to large Ags but no change in those to small Ags.

\section{DETERMINATION OF STOICHIOMETRY}

\subsection{Data Analysis}

The apparent stoichiometry of the surface complex (i.e. the number of $\mathrm{Ag}$ molecules that can bind to one $\mathrm{Ab}$ molecule) was calculated from the saturating binding capacity of the surface by the equation (Tobita et al., 2004; Oda et al., 2006):

stoichiometry $\mathrm{n}=\frac{R_{\mathrm{Ag}}}{R_{\mathrm{Ab}}} \times \frac{\mathrm{MW} \mathrm{Ab}}{\mathrm{MW} \mathrm{Ag}}=\frac{\mathrm{RU}_{\max }-\mathrm{RU}_{\mathrm{i}}}{\mathrm{RU}_{\mathrm{i}}-\mathrm{RU}_{0}} \times \frac{\mathrm{MW} \mathrm{Ab}}{\mathrm{MW} \mathrm{Ag}}$

where $R_{\mathrm{Ab}}$ is the SPR response of surface immobilized Ab in RUs. $R_{\mathrm{Ag}}$ is the observed response of bound antigen. Relationship between bound $\mathrm{Ag}\left(\mathrm{RU}_{\max }-\mathrm{RU}_{\mathrm{i}}\right)$ and amount of $\mathrm{Ab}\left(\mathrm{RU}_{\mathrm{i}}-\mathrm{RU}_{\mathrm{o}}\right)$ present on the sensor surface is displayed in (Fig. 7).

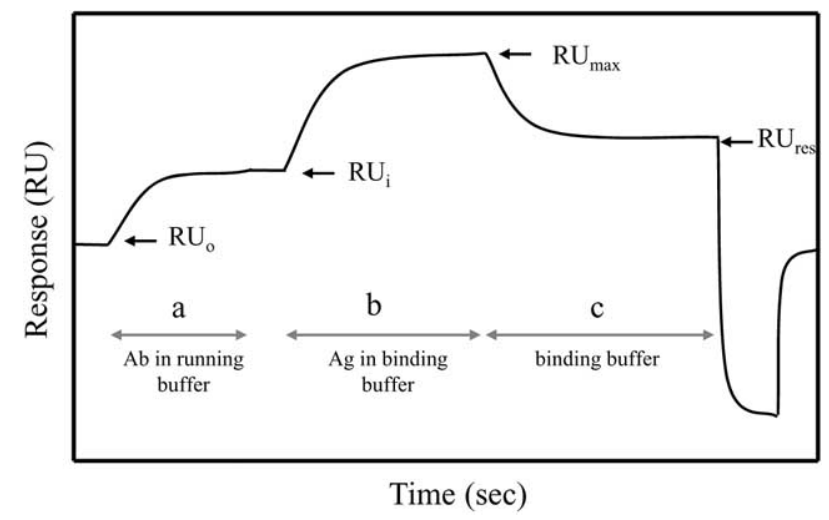

Fig. (7). A simple simulated sensorgram illustrating the binding of an $\mathrm{Ag}$ to an $\mathrm{Ab}$ immobilized on a sensor chip. A running buffer solution is pumped at a constant flow rate over the sensor surface. The sensorgram shows that injection of $\mathrm{Ab}$ generates an increase in the response expressed as resonance units (RU) $\left(R U_{\mathrm{i}}-R U_{\mathrm{o}}\right)$ as shown in a. A pulse of sample containing the $\mathrm{Ag}$ is then injected and association of the $\mathrm{Ag}$ to the $\mathrm{Ab}$ is shown in $\mathrm{b}$. An increase $\left(R U_{\max }-R U_{i}\right)$ of resonance unit is observed following an injection of a sample. The decrease of RU during the washing step (injection of binding buffer) is shown in c.

\subsection{Example}

Oda and co-workers (2006) investigated the interaction of Abs with protein Ags of different size, such as HEL, ovalbumin (OVA), and BSA, using SPR in order to estimate regional and segmental $\mathrm{Ab}$ flexibility. When Abs were immobilized on the surface of a sensor chip through the $\mathrm{Fc}$ region, the stoichiometry of the $\mathrm{Ag}-\mathrm{Ab}$ complex was dependent on the $\mathrm{Ag}$ size; $\mathrm{Ag}_{2} \mathrm{Ab}_{1}$ forming with $\mathrm{HEL}$ and $\mathrm{Ag}_{1} \mathrm{Ab}_{1}$ with OVA and BSA. Typical sensorgrams for the bind- 
A

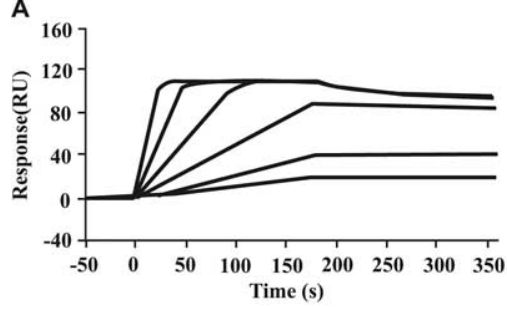

C
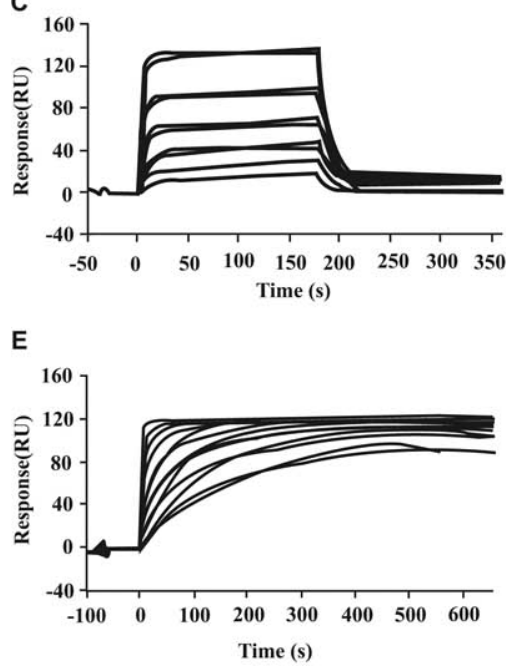

B

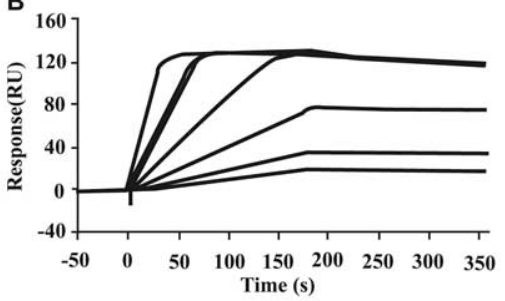

D
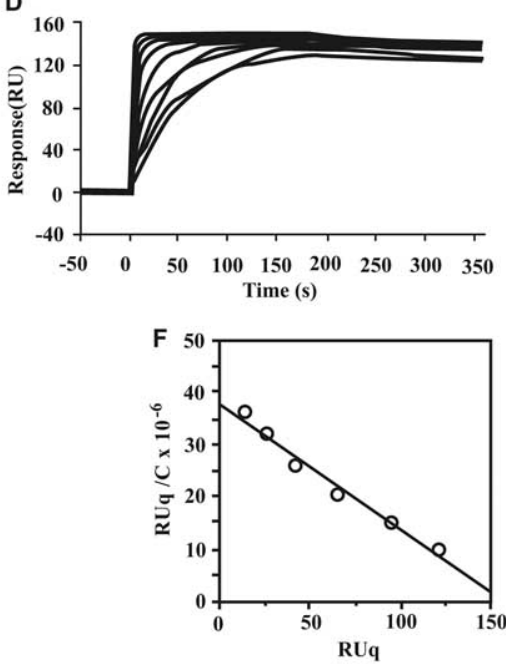

Fig. (8). SPR analysis of interactions between Ags and immobilized mAbs. (A-E) Sensorgrams for the interactions between HEL and HC1 anti-HEL mAb (A), between HEL and HC2 anti-HEL mAb (B), between OVA and O1-1 anti-OVA mAb (C), between OVA and YOVA anti-OVA mAb (D), and between BSA and KM10 anti-BSA mAb (E). Measurements were made at a concentration range of 0.78-25 nM for HEL, 400-12 800 nM for OVA, and 25-1600 nM for BSA. Sensorgrams (solid black lines) were subjected to nonlinear fitting (dotted grey line) to estimate the $k_{\mathrm{a}}$ and $k_{\mathrm{d}}$ values. The amounts of $\mathrm{mAb}$ immobilized by RAMFc Ab on the CM5 sensor chip were 650 RU of HC1, 650 RU of HC2, 608 RU of O1-1, 490 RU of YOVA, and 243 RU of KM10, respectively. (F) Scatchard plots for OVA binding to immobilized O1-1 anti-OVA mAb at a concentration of 400-12 800 nM. Adapted with permission from reference (Oda et al., 2006).

ing of the $\mathrm{Ag}$ to the immobilized mAbs on the CM5 chip were shown in Fig. 8A-E. In the case of OVA and O1-1, the interaction was evaluated using Scatchard plot analysis (Fig. 8F), because the reaction proceeded rapidly and attained equilibrium within the measurement time. The plot was linear with a $K$ a value of $2.4 \times 10^{5}$ $\mathrm{M}^{-1}$. Binding stoichiometries for the respective $\mathrm{Ag}-\mathrm{mAb}$ interactions were 1.8 for $\mathrm{HC} 1$ anti-HEL mAb, 2.1 for HC2 anti-HEL mAb,

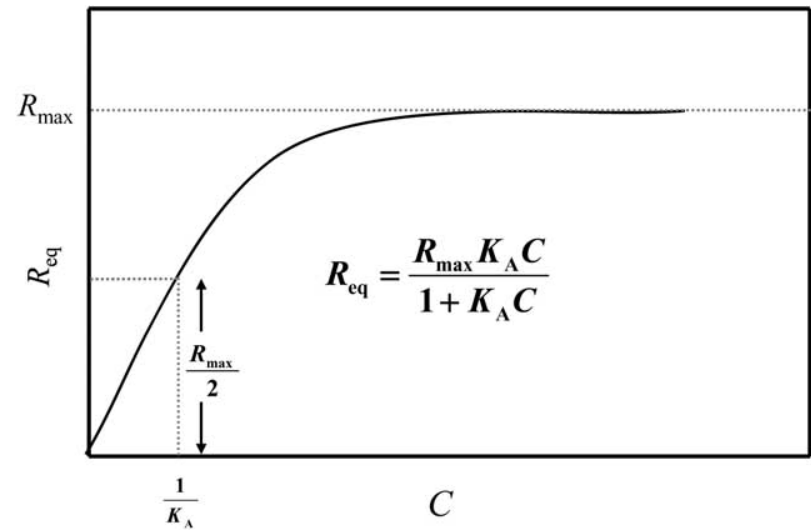

Fig. (9). A binding curve of the steady state binding response $R_{\mathrm{eq}}$ versus $\mathrm{Ag}$ concentration $C$ according to Eq. [15].

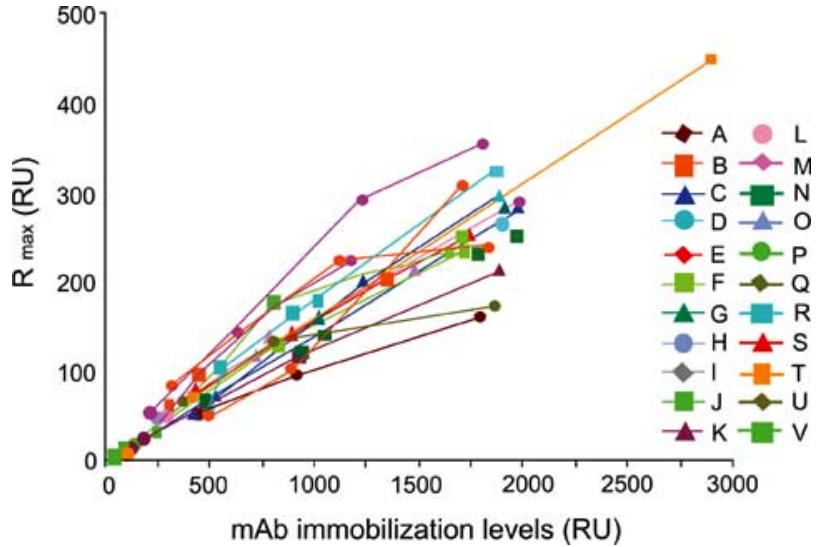

Fig. (10). Relationship between $R_{\max }$ and mAb immobilization levels $R_{\mathrm{Ab}}$. The $R_{\max }$ calculated for each data set was plotted against the levels of mAb immobilized on multiple surfaces by the 22 participants (A-V). Reprinted with permission from reference (Katsamba et al., 2006).

0.9 for O1-1 anti-OVA mAb, 1.0 for YOVA anti-OVA mAb and 1.1 for KM10 anti-BSA mAb. The results clearly show that an antiHEL mAb can bind two HEL molecules simultaneously, whereas 
anti-OVA or anti-BSA mAbs can bind only a single molecule of OVA or BSA, respectively. The results obtained allowed them to propose that the Fab arms of B-cell receptors whose Fc regions are immobilized on cell surface have a reduced dynamic range.

\section{DETERMINATION OF MAXIMUM BINDING RATIO}

\subsection{Data Analysis}

The measured SPR response in resonance units (RUs) is assumed to correlate to the surface concentration of immobilized $\mathrm{Ab}$ and $\mathrm{Ag}$ bound using the estimate $1 \mathrm{RU} \approx 1 \mathrm{pg} \mathrm{mm}^{-2}$ as has been done in the past for proteins (Yang et al., 2005). Because of this, the maximum binding capacity or $R_{\max }$ value can be used to calculate a maximum binding ratio ( $\mathrm{mol} \mathrm{Ag}$ bound/mol $\mathrm{Ab}$ immobilized), assuming one set of independent noninteracting binding sites, using the following equation:

$$
\text { maximum binding ratio }=\frac{\mathrm{R}_{\max }}{\mathrm{R}_{\mathrm{Ab}}} \times \frac{\mathrm{MW} \mathrm{Ab}}{\mathrm{MW} \mathrm{Ag}} \times \frac{1}{n}
$$

where $R_{\mathrm{Ab}}$ is the amount of surface immobilized Ab in RUs and $n$ is the number of binding sites on the $\mathrm{Ab} . R_{\max }$ is the maximum response at saturation of surface binding sites and can be determined by the method described below.

At equilibrium, $\mathrm{d} R / \mathrm{d} t=0$ and Eq. [3] can be rearranged as follows:

$$
R_{\mathrm{eq}}=\left(R_{\mathrm{max}} K_{\mathrm{A}} C\right) /\left(1+K_{\mathrm{A}} C\right)
$$

where $C$ is the concentration of Ag injected, $K_{\mathrm{A}}$ is the apparent association constant. $R_{\text {eq }}$ is the measured response at equilibrium, and values of $R_{\text {eq }}$ are obtained at a series of injected analyte $C$ solution concentrations. The steady state response can be plotted versus the analyte concentration and the data are fit to a simple Langmuir isotherm for molecular interactions (Fig. 9). $R_{\max }$ can then be calculated from the best fit of the binding curve.

Thus, the maximum binding ratio (Ag molecules per $\mathrm{Ab}$ ) can be calculated by Eq. [14] using the $R_{\max }$ obtained by Eq. [15] and the known amount of $\mathrm{Ab}$ immobilized.

\subsection{Examples}

Katsamba and co-workers (2006) measured the binding of prostate-specific antigen (PSA) to a monoclonal anti-PSA antibody $(\mathrm{mAb})$ to explore the reliability of Biacore-based SPR immunosensing. The $\mathrm{mAb}$ was immobilized on the sensor chip at three different densities and a two-step assay was used to determine the kinetic and affinity parameters of the PSA/mAb complex. The association and the extended-dissociation data derived from the three antibody surfaces were globally fitted using a simple 1:1 interaction model. The average $k_{\mathrm{a}}$ and $k_{\mathrm{d}}$ for the PSA/mAb interaction as calculated from the 22 analyses were $(4.1 \pm 0.6) \times 10^{4} \mathrm{M}^{-1} \mathrm{~s}^{-1}$ and $(4.5 \pm 0.6) \times 10^{-5} \mathrm{~s}^{-}$ ${ }^{1}$, respectively. Based on the kinetic rate constants, the affinity $\left(K_{\mathrm{D}}\right)$ of the PSA/mAb interaction was $1.1 \pm 0.2 \mathrm{nM}$. Fig. (10) shows the $\mathrm{mAb}$ immobilization level plotted against the $R_{\max }$ obtained from fitting the antigen binding response. As expected, this plot shows a nearly linear correlation between the level of $\mathrm{mAb}$ immobilized and the $R_{\max }$. As the amount of immobilized mAb changes, the number of $\mathrm{mAb}$ binding sites available for interaction changes proportionally. Moreover, the ratio of the molecular masses of the reactants and the immobilized and observed response levels were used to obtain information about the activity of the surface ligand. In this work, the mass of the mAb is $75 \mathrm{kDa}$ per antigen binding site and the mass of monomeric PSA is $30 \mathrm{kDa}$, giving a mass ratio of 0.40 . The average value of the observed $R_{\max }$ vs immobilization level $R_{\mathrm{Ab}}$ (slope of the data in Fig. 10) was 0.175. The difference between the observed response and the expected response based on the mass ratio was 0.4375 (i.e., maximum binding ratio). This suggested that the $\mathrm{mAb}$ surface was only $44 \%$ active for PSA binding.

\section{CONCLUSION}

The SPR techniques can be used at two levels of biomolecular interaction analysis. Firstly, in providing simple yes/no answers to the question: Does Ag bind to Ab? Secondly, they can, with care, provide accurate binding affinity and stoichiometry data. Both methods are sensitive to the binding of mass to an activated optical sensor chip surface. The literatures surveyed in this review demonstrate that SPR can successfully detect and quantitate binding of small molecule of hapten to $\mathrm{Ab}$ by three different interaction modes: nonequivalent two-site interaction, multiple equivalent-site interaction and multisite interaction modes. A variety of SPR approaches have been devised as described in this review for obtaining an estimate of the binding constant of an Ab for its Ag. Many immunochemical procedures involve multivalent interactions. Antibodies are multivalent; IgGs and most IgAs are bivalent, and IgMs are decavalent. Antigens can be multivalent either because they contain multiple copies of the same epitope, or because they contain multiple epitopes recognized by different Abs. It is not always easy to predict the effects of multivalency, as the reactions involve geometric arrangements that impose steric constraints. However, the effects of multivalency can be simplified by the use of SPR by considering several simple modes with a 1:1 or 1:2 stoichiometry. On the other hand, SPR has been used to evaluate the intrinsic affinities and rate constants for binding of the Ag molecules to $\mathrm{Ab}$. However, to date there is no proof of concept that SPR sensing can be performed for defining exactly how many subpopulations of different affinity exist in a polyclonal $\mathrm{Ab}$ which contains a heterogeneous population of Abs of different affinity for the immunogen. Nevertheless, SPR sensing is emerging as a method of great value for the measurement of binding constant, dissociation rate constant and stoichimetry for $\mathrm{Ab}-\mathrm{Ag}$ interaction kinetics in the emerging fields of genomics, proteomics and metabolomics.

\section{ABBREVIATIONS}

$\begin{array}{ll}\mathrm{Ag} & =\text { Antigen } \\ \mathrm{BSA} & =\text { Bovine serum albumin } \\ \mathrm{DHP} & =\text { Dihydroprogesterone } \\ \mathrm{Fab} & =\text { Antigen-binding fragment } \\ \mathrm{HEL} & =\text { Hen egg white lysozyme } \\ K_{\mathrm{A}} & =\text { Association constant } \\ k_{\mathrm{a}} & =\text { Association rate constant } \\ K_{\mathrm{D}} & =\text { Dissociation constant } \\ k_{\mathrm{d}} & =\text { Dissociation rate constant } \\ \mathrm{mAb} & =\text { Monoclonal antibody } \\ \mathrm{NP} & =\text { 4-hydroxy-3-nitrophenyl)acetyl } \\ \mathrm{OVA} & =\text { Ovalbumin } \\ \mathrm{PSA} & =\text { Prostate-specific antigen } \\ R & =\text { SPR response signal } \\ \mathrm{RU} & =\text { Resonance unit } \\ \mathrm{scFv} & =\text { Variable single-chain fragment } \\ \mathrm{SPR} & =\text { Surface plasmon resonance }\end{array}$

\section{REFERENCES}

Beernink, P. T., Hwang, M., Ramirez, M., Murphy, M. B., Doyle, S. A. and Thelen, M. P. (2005) Specificity of protein interactions mediated by BRCT domains of the XRCC1 DNA repair protein. J. Biol. Chem. 280: 30206-13.

Berzofsky, J. A. and Berkower, I. J. (1983) in: W.E. Paul (Ed.) Antibodyantigen interaction. CRC Press, Boca Raton, FL., pp. 595-644.

Biemann, H. P. and Koshland, D. E. (1994) The proteinase: mucus proteinase inhibitor binding stoichiometry. J. Biol. Chem. 267: 4370-5.

Biorn, A. C., Cocklin, S., Madani, N., Si, Z., Ivanovic, T., Samanen, J., Ryk, D. I. V., Pantophlet, R., et al. (2004) Model of action for linear peptide inhibitors of HIV-1 gp120 interactions. Biochemistry 43: 1928-38. 
Boghaert, E. R., Sridharan, L., Armellino, D. C., Khandke, K. M., DiJoseph, J. F., Kunz, A., Dougher, M. M., Jiang, F., et al. (2004) Antibodytargeted chemotherapy with the calicheamicin conjugate hu3S193-Nacetyl $\mathrm{g}$ calicheamicin dimethyl hydrazide targets Lewis and eliminates Lewis-positive human carcinoma cells and xenografts. Clin. Cancer Res. 10: 4538-49.

Canziani, G. A., Klakamp, S. and Myszka, D. G. (2004) Kinetics screening of antibodies from crude hybridoma samples using Biacore. Anal. Biochem. 325: 301-7.

Carrasco, C., Facompré, M., Chisholm, J. D., Vranken, D. L. V., Wilson, W. D. and Bailly, C. (2002) Nucleic Acids Res. 30: 1774-81.

Chaudhury, C., Brooks, C. L., Carter, D. C., Robinson, J. M. and Anderson, C. L. (2006) Albumin binding to FcRn: Distinct from the FcRn-IgG interaction. Biochemistry 45: 4983-90.

Davies, J. (1994) Review:Surface plasmon resonance-The technique and its applications to biomaterial processes. Nanobiology 3: 5-16.

Djavadi-Ohaniance, L. and Friguet, B. (1992) The immunochemistry of solid-phase immunoassay. CRC Press, Boca Raton, FL, pp. 201-6.

Drake, A. W., Myszka, D. G. and Klakamp, S. L. (2004) Characterizing high-affinity antigen/antibody complexes by kinetic- and equilibriumbased methods. Anal. Biochem. 328: 35-43.

Dubreuil, O., Bossus, M., Graille, M., Bilous, M., Savatier, A., Jolivet, M., Ménez, A., Stura, E., et al. (2005) Fine tuning of the specificity of an anti-progesterone antibody by first and second sphere residue engineering. J. Biol. Chem. 280: 24880-7.

Fountoulakis, M., Zulauf, M., Lustig, A. and Garotta, G. (1992) Stoichiometry of interaction between interferon gamma and its receptor. Eur. J. Biochem. 208: 781-7.

Glaser, R.W. (1993) Antigen-antibody binding and mass transport by convection and diffusion to a surface: a two-dimensional computer model of binding and dissociation kinetics. Anal. Biochem. 213: 152-61.

Goldberg, M. (1991) Investigating protein conformation, dynamics and folding with monoclonal antibodies. TIBS 16: 358-62.

Gomes, P. and Andreu, D. (2002) Direct kinetic assay of interactions between small peptides and immobilized antibodies using a surface plasmon resonance biosensor. J. Immunol. Methods 259: 217-30.

Herr, A. B., White, C. L., Milburn, C., Wu, C. and Bjorkman, P. J. (2003) Bivalent binding of $\operatorname{IgA} 1$ to FcaRI suggests a mechanism for cytokine activation of IgA phagocytosis. J. Mol. Biol. 327: 645-57.

Huang, B. C., Davern, S. and Kennel, S. J. (2006) Mono and bivalent binding of $\mathrm{scFv}$ and covalent diabody to murine laminin-1 using radioiodinated proteins and SPR measurements: effects on tissue retention in vivo. J. Immunol. Methods 313: 149-60.

Ja, W. W., Olsen, B. N. and Roberts, R. W. (2005) Epitope mapping using mRNA display and a unidirectional nested deletion library. Protein Eng. Des. Sel. 18: 309-19.

Johansson, R., Ohlin, M., Jansson, B. and Ohlson, S. (2006) Transiently binding antibody fragments against Lewis $\mathrm{x}$ and sialyl-Lewis $\mathrm{x}$. J. Immunol. Methods 312: 20-6.

Jonsson, U. (1992) Real time BIA. A new biosensor based technology for the direct measurement of biomolecular interactions. Biosensors: Fundamentals, Technologies and Applications. Ed. Scheller F. and Schmid R. D., pp. 467-476.

Katsamba, P. S., Navratilova, I., Calderon-Cacia, M., Fan, L., Thornton, K., Zhu, M., Bos, T. V., Forte, C., et al. (2006) Kinetic analysis of a highaffinity antibody/antigen interaction performed by multiple Biacore users. Anal. Biochem. 352: 208-21.

Lackmann, M., Bucci, T., Mann, R. J., Kravets, L. A., Viney, E., Smith, F., Moritz, R. L., Carter, W., et al. (1996) Purification of a ligand for the EPH-like receptor HEK using a biosensor-based affinity detection approach. Proc. Natl. Acad. Sci. USA 93: 2523-7.

Leickt, L., Grubb, A. and Ohlson, S. (2002) Development of monoclonal antibodies against creatine kinase MB2. Scand J. Clin. Lab. Invest. 62 : 423-30.

Lin, L.-P., Huang, L.-S., Lin, C.-W., Lee, C.-K., Chen, J.-L., Hsu, S.-M. and Lin, S. (2005) Determination of binding constant of DNA-binding drug to target DNA by surface plasmon resonance biosensor technology. Curr. Drug Targets 5: 61-72.

Liu, X., Wei, J., Song, D., Zhang, Z., Zhang, H. and Luo, G. (2003) Determination of affinities and antigenic epitopes of bovine cardiac troponin I (cTnI) with monoclonal antibodies by surface plasmon resonance biosensor. Anal. Biochem. 314: 301-9.

Liu, Z., Li, F. and Huang, Y. (1999) Determination of unbound drug concentration and protein-drug binding fraction in plasma. Biomed. Chro- matogr. 13: 262-6.

Ma, Q., Shimaoka, M., Lu, C., Jing, H., Carman, C. V. and Springer, T. A. (2002) Activation-induced conformational changes in the I domain region of lymphocyte function-associated antigen 1. J. Biol. Chem. 277: 10638-41.

Markey, F. (1999) What is SPR anyway? BIA. J. 6: 14-7.

Markgren, P.-O., Hämäläinen, M. and Danielson, U. (1999) Screening of compounds interacting with HIV-1 proteinase using optical biosensor technology. Anal. Biochem. 265: 340-50.

Mazur, S., Tanious, F. A., Ding, D., Kumar, A., Boykin, D. W., Simpson, I. J., Neidle, S. and Wilson, W. D. (2000) A thermodynamic and structural analysis of DNA minor-groove complex formation. J. Mol. Biol. 300: 321-37.

Mimura, Y., Sondermann, P., Ghirlando, R., Lund, J., Young, S. P., Goodall, M. and Jefferis, R. (2001) Role of oligosaccharide residues of IgG1-Fc in FccRIIb binding. J. Biol. Chem. 276: 45539-45547.

Morton, T. A. and Myszka, D. G. (1998) Kinetic analysis of macromolecular interactions using surface plasmon resonance biosensors. Methods Enzymol. 295: 268-94.

Myszka, D. G. (1999) Survey of the 1998 optical biosensor literature. J. Mol. Recognit. 12: 390-408.

Myszka, D. G., Jonsen, M. D. and Graves, B. J. (1998) Equilibrium analysis of high affinity interactions using BIACORE. Anal. Biochem. 265: 32630 .

Nguyen, B., Tardy, C., Bailly, C., Colson, P., Houssier, C., Kumar, A., Boykin, D. W. and Wilson, W. D. (2002) Influence of compound structure on affinity, sequence selectivity, and mode of binding to DNA for unfused aromatic dications related to furamidine. Biopolymers 63: 28197.

Oda, M. and Azuma T. (2000) Reevaluation of stoichiometry and affinity/avidity in interactions between anti-hapten antibodies and mono- or multi-valent antigens. Mol. Immunol. 37: 1111-22.

Oda, M., Uchiyama, S., Robinson, C. V., Fukui, K., Kobayashi, Y. and Azuma, T. (2006) Regional and segmental flexibility of antibodies in interaction with antigens of different size. FEBS J. 273: 1476-87.

Okun, V. M., Moser, R., Blaas, D. and Kenndler, E. (2001) Complexes between monoclonal antibodies and receptor fragments with a common cold virus: determination of stoichiometry by capillary electrophoresis. Anal. Chem. 73: 3900-6.

Paetz, A., Sack, M., Thepen, T., Tur, M. K., Bruell, D., Finnern, R., Fischer, R. and Barth, S. (2005) Recombinant soluble human Fcg receptor I with picomolar affinity for immunoglobulin G. Biochem. Biophys. Res. Commun. 338: 1811-7.

Ricard-Blum, S., Bernocco, S., Font, B., Moali, C. Eichenberger, D., Farjane, J., Burchardt, E. R., van der Rest, M., et al. (2002) Interaction properties of the procollagen C-proteinase enhancer protein shed light on the mechanism of stimulation of BMP-1. J. Biol. Chem. 277: 338649.

Rich, R. L. and Myszka, D. G. (2000) Advances in surface plasmon resonance biosensor analysis. Curr. Opin. Biotech. 11: 54-61.

Roos, H., Karlsson, R., Nilshans, H. and Persson, A. (1998) Thermodynamic analysis of protein interactions with biosensor technology. $J$. Mol. Recognit. 11: 204-10.

Russo, K., Ragone, R., Facchiano, A. M., Capogrossi, M. C. and Facchiano, A. (2002) Platelet-derived growth factor-BB abd basic fibroblast growth factor directly interact in vitro with high affinity. J. Biol. Chem. 277: 1284-91.

Sagawa, T., Oda, M., Ishimura, M., Furukawa, K. and Azuma, T. (2003) Thermodynamic and kinetic aspects of antibody evolution during the immune response to hapten. Mol. Immunol. 39: 801-8.

Son, J. H., Lee, U. H., Lee, J. J., Kwon, B., Kwon, B. S. and Park, J. W. (2004) Humanization of agonistic anti-human 4-1BB monoclonal antibody using a phag-displayed combinatorial library. J. Immunol. Methods 286: 187-201.

Teulade-Fichou, M. P., Carrasco, C., Guittat, L., Bailly, C., Alberti, P., Mergny, J. L., David, A., Lehn, J. M., et al. (2003) Selective recognition of G-qQuadruplex telomeric DNA by a bis(quinacridine) macrocycle. J. Am. Chem. Soc. 125: 4732-40.

Tobita, T., Oda, M. and Azuma, T. (2004) Segmental flexibility and avidity of IgM in the interaction of polyvalent antigens. Mol. Immunol. 40: 803-11.

Verel, I., Heider, K. H., Siegmund, M., Ostermann, E., Patzelt, E., Sproll, M., Snow, G. B., Adolf, G. R., et al. (2002) Tumor targeting properties of monoclonal antibodies with different affinity for target antigen 
CD44V6 in nude mice bearing head-and-neck cancer xenografts. Int. J. Cancer 99: 396-402.

Wittel, U. A., Jain, M., Goel, A., Baranowska-Kortylewicz, J., Kurizaki, T., Chauhan, S. C., Agrawal, D. K., Colcher, D., et al. (2005) Engineering and characterization of a divalent single-chain Fv angiotensin II fusion contruct of the monoclonal antibody CC49. Biochem. Biophys. Res. Commun. 329: 168-76.
Yang, C. Y., Brooks, E., Li, Y., Denny, P., Ho, C. M., Qi, F., Shi, W., Wolinsky, L., et al. (2005) Detection of picomolar levels of interleukin8 in human saliva by SPR. Lab Chip 5: 1017-23.

Zeder-Lutz, G., Neurath, A. R. and Van Regenmortel, M. H. (1999) Kinetics of interaction between 3-hydroxyphthaloyl-beta-lactoglobulin and CD4 molecules. Biologicals 27: 29-34. 
Copyright of Current Proteomics is the property of Bentham Science Publishers Ltd. and its content may not be copied or emailed to multiple sites or posted to a listserv without the copyright holder's express written permission. However, users may print, download, or email articles for individual use. 